\title{
FTH1 Inhibits Ferroptosis Through Ferritinophagy in the 6-OHDA Model of Parkinson's Disease
}

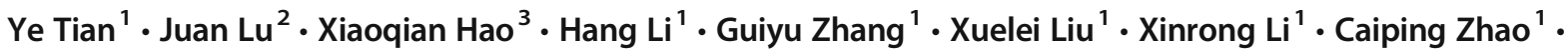

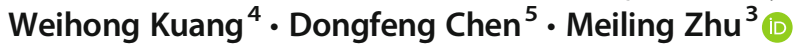

Accepted: 4 September 2020 / Published online: 21 September 2020

(C) The American Society for Experimental NeuroTherapeutics, Inc. 2020

\begin{abstract}
Parkinson's disease (PD) is a neurodegenerative disorder characterized by degeneration of dopaminergic neurons associated with dysregulation of iron homeostasis in the brain. Ferroptosis is an iron-dependent cell death process that serves as a significant regulatory mechanism in PD. However, its underlying mechanisms are not yet fully understood. By performing RNA sequencing analysis, we found that the main iron storage protein ferritin heavy chain 1 (FTH1) is differentially expressed in the rat 6hydroyxdopamine (6-OHDA) model of PD compared with control rats. Our present work demonstrates that FTH1 is involved in iron accumulation and the ferroptosis pathway in this model. Knockdown of FTH1 in PC-12 cells significantly inhibited cell viability and caused mitochondrial dysfunction. Moreover, FTH1 was found to be involved in ferritinophagy, a selective form of autophagy involving the degradation of ferritin by ferroptosis. Overexpression of FTH1 in PC-12 cells impaired ferritinophagy and downregulated microtubule-associated protein light chain 3 and nuclear receptor coactivator 4 expression, ultimately suppressing cell death induced by ferroptosis. Consistent with these findings, the ferritinophagy inhibitors chloroquine and bafilomycin A1 inhibited ferritin degradation and ferroptosis in 6-OHDA-treated PC-12 cells. This entire process was mediated by the cyclic regulation of FTH1 and ferritinophagy. Taken together, these results suggest that FTH1 links ferritinophagy and ferroptosis in the 6-OHDA model of $\mathrm{PD}$, and provide a new perspective and potential for a pharmacological target in this disease.
\end{abstract}

Key Words FTH1 $\cdot$ ferritinophagy $\cdot$ ferroptosis $\cdot$ iron $\cdot$ Parkinson's disease.

Electronic supplementary material The online version of this article (https://doi.org/10.1007/s13311-020-00929-z) contains supplementary material, which is available to authorized users.

Dongfeng Chen

cdf27212@21cn.com

Meiling Zhu

meilingzhubazyy@126.com

1 Shenzhen Bao'an Traditional Chinese Medicine Hospital (Group), Guangzhou University of Chinese Medicine, Shenzhen 518133, China

2 Shenzhen Hospital of Southern Medical University, Shenzhen 518000, China

3 Shenzhen Hospital of Integrated Traditional Chinese and Western Medicine, Guangzhou University of Chinese Medicine, Shenzhen 518104, China

4 The Second Clinical Medical College, Guangdong Medical University, Dongguan 524023, China

5 The Research Center of Basic Integrative Medicine, Guangzhou University of Chinese Medicine, Guangzhou 510405, China

\section{Introduction}

Parkinson's disease (PD) is the second most common chronic neurodegenerative disease and is characterized by the formation of Lewy bodies and loss of dopaminergic neurons in the substantia nigra (SN) [1,2]. Dyskinesia and cognitive impairment seriously reduce the quality of life of patients with PD. PD has not yet been cured, and medications can only improve the symptoms $[3,4]$. Therefore, there is an urgent need to develop novel targets to improve therapeutic and diagnostic approaches for PD patients.

Iron metabolism plays a critical role in the pathogenesis of neurodegenerative diseases, and iron has been identified as a potential target for PD $[5,6]$. Iron is vital for cell proliferation and survival. Conversely, excessive accumulation of iron increases the oxidation of dopamine and induces the loss of dopaminergic neurons in PD [7, 8]. Relevant research has shown that ferroptosis is involved in the pathogenesis of PD. Ferroptosis is an iron-dependent lipid peroxidation process that is related to cell death; it is associated with a decrease in 
glutathione peroxidase 4 (GPX4) activity and accumulation of reactive oxygen species on membrane lipids [9-12]. However, the underlying mechanism of ferroptosis in PD has not been fully clarified.

Ferroptosis was previously thought to be independent of autophagy, but recent studies have shown that ferroptosis is a form of autophagic cell death involving the degradation of ferritin [13-15]. In this study, we found that ferritin heavy chain 1 (FTH1) was differentially expressed in PD model rats compared with control rats by RNA sequencing analysis. FTH1 plays an important role in the maintenance of the cellular iron balance in ferroptosis. Interestingly, FTH1 was also found to be involved in ferritinophagy, a selective form of autophagy. Ferritinophagy is a process involving the autophagic degradation of ferritin, and it provides a substrate for ferroptosis [16-18]. The process is mediated by nuclear receptor coactivator 4 (NCOA4), which selectively binds FTH1 in the autophagosome and delivers it into the lysosome, resulting in iron release [19-21]. High concentrations of iron inhibit the binding of FTH1 to NCOA4 and enhance the degradation of NCOA4 $[16,22]$. This process leads to the inhibition of ferritin degradation and ferroptosis, indicating that ferroptosis is an autophagic cell death process [18]. Notably, the role of ferritinophagy and the link between ferritinophagy and ferroptosis in PD have not been explored and are worthy of further study. In this study, we investigated the role of FTH1 and its effects in ferroptosis and ferritinophagy. Our results confirmed that FTH1 increases cell viability and links ferritinophagy to ferroptosis in the 6-OHDA model of PD. Moreover, the data suggested that FTH1 inducers may be a pharmacological target for PD.

\section{Materials and Methods}

\section{Animal Studies}

Adult male Sprague-Dawley rats were used according to protocols approved by the Animal Care and Use Committee at the Peking University Shenzhen Graduate School (Shenzhen, China) and provided free access to food and water. The rats were divided into 3 groups (the normal, sham, and model groups) with 12 rats in each group. To establish a rat model of PD, 6-OHDA was injected into the right $\mathrm{SN}$ and ventral tegmental area of each at. Animal surgery was performed as previously reported [23].

\section{HE Staining}

Rats were anesthetized with $10 \%$ chloral hydrate $(4 \mathrm{ml} / \mathrm{kg})$. Brain tissues were fixed in $4 \%$ paraformaldehyde in phosphate buffer $(\mathrm{pH}=7.4)$. Paraffin-embedded tissues were sectioned, deparaffinized, and hydrated. All slides were stained with hematoxylin or hematoxylin and eosin and imaged using light microscopy.

\section{Immunohistochemistry}

Paraffin sections were dewaxed, soaked in xylene for $15 \mathrm{~min}$, and hydrated with ethanol (anhydrous ethanol, 95\% ethanol, $90 \%$ ethanol, and $70 \%$ ethanol for $10 \mathrm{~min}$ each). The sections were blocked in goat serum at room temperature for $20 \mathrm{~min}$. Then, the sections were washed 3 times in PBS for 5 min each and blocked with $5 \%$ bovine serum albumin. After blocking, sections were incubated with FTH1 (1:100, Cell Signaling Technology, MA, USA), tyrosine hydroxylase (TH) (1:100, Sigma-Aldrich, MO, USA), and $\alpha$-synuclein ( $\alpha$-syn) (1:100, Cell Signaling Technology) antibodies overnight. The sections were washed 3 times in PBS and incubated with secondary antibodies (1:5000, EARTH, CA, USA) in an incubator at room temperature for $1 \mathrm{~h}$. The tissue sections were incubated with diaminobenzidine substrate solution (BOSTER, Wuhan, China).

\section{RNA Sequence Analysis}

RNA sequencing was performed on the BGISEQ-500 platform (BGI-Shenzhen, China). RNA was isolated from 6 tissue samples, namely 3 from the PD model group and 3 from the normal group. The data were visualized by using the Dr.Tom system provided by BGI. RNA sequencing was performed via a random sampling process. To improve the accuracy of the differentially expressed gene results, a gene was considered a differentially expressed gene when the fold change was $\geq 2$ and the $Q$ value was $\leq 0.001$.

\section{Cell Culture and Reagents}

Rattus norvegicus PC-12 cells were obtained from Stem Cell Bank, Chinese Academy of Sciences. The cells were maintained in RPMI 1640 medium (Gibco, CA, USA) supplemented with $10 \%$ fetal bovine serum (Gibco). 6-OHDA was purchased from Sigma-Aldrich. Erastin, deferoxamine (DFO), ferrostatin-1 (Fer-1), chloroquine (CQ), and bafilomycin A1 (Baf-A1) were obtained from MedChem Express (NJ, USA).

\section{Cell Viability Assay}

Cell viability was evaluated using an MTS assay kit (Promega, WI, USA). Briefly, PC-12 cells $\left(5 \times 10^{3} /\right.$ well $)$ were seeded in flat-bottomed 96-well plates. Then, the original cell culture medium was discarded, MTS was added to each well, and the cells were incubated for another $2 \mathrm{~h}$. The OD value was recorded with a microplate reader (BioTek, VT, USA) at a wavelength of $490 \mathrm{~nm}$. The data are expressed as means \pm 
S.D., and the $n$ was $\geq 3$ per group for all the studies ( $t$ test, $* p<0.05, * * p<0.01, * * * p<0.001)$.

Living and dead cells were detected using a calcein-AM/PI assay kit (Solarbio, Beijing, China). PC-12 cells $\left(5 \times 10^{3} /\right.$ well) were seeded in 96-well black-walled multiwell plates. After overnight culture, the cells were treated with various concentrations of 6-OHDA for $24 \mathrm{~h}$. The cells were then washed twice with buffer and treated with $5 \mu \mathrm{M}$ PI (to stain dead cells, red) and $5 \mu \mathrm{M}$ calcein-AM (to stain living cells, green) for $20 \mathrm{~min}$. The cells were observed with a fluorescence microscope (Olympus, Tokyo, Japan).

\section{Western Blot Analysis}

Cells were harvested by centrifugation at $12,000 \mathrm{rpm}$ for 15 min at $4{ }^{\circ} \mathrm{C}$ and lysed in RIPA buffer (Sigma-Aldrich). Protein concentrations were determined using a BCA protein assay kit (Thermo Scientific, CA, USA). After boiling for $10 \mathrm{~min}$ in loading buffer, the samples ( $20 \mu \mathrm{g}$ protein per lane) were separated by $10 \%$ or $12 \%$ SDS-PAGE. The proteins were transferred to nitrocellulose membranes with $0.45-\mu \mathrm{m}$ diameter pores. The membranes were blocked with $5 \%$ nonfat dry milk for $1 \mathrm{~h}$ at room temperature and then incubated with primary antibodies, including FTH1 (1:1000, Cell Signaling Technology), GPX4 (1:1000, Cell Signaling Technology), $\beta$ tubulin (1:1000, Cell Signaling Technology), TH (1:1000, Cell Signaling Technology; 1:500, Sigma-Aldrich), $\alpha$-syn (1:1000, Cell Signaling Technology), NCOA4 (ARA70, 1:500, Santa Cruz Biotechnology, CA, USA), and light chain 3 (LC3)B (1:1000, Abcam, Cambridge, UK) antibodies. After incubation overnight, the membranes were washed 4 times in TBST and incubated with secondary antibody (1:1000; Cell Signaling Technology) for another $1 \mathrm{~h}$ at room temperature. The protein bands were visualized with Immobilon Western HRP Substrate (Millipore, MA, USA). Densitometric analyses of the bands were performed using ImageJ software. All data are representative of at least 3 independent experiments.

\section{Cell Transfection}

siRNAs targeting FTH1 were designed and synthesized by GenePharma (Shanghai, China). The sequences of the siRNAs were as follows: siRNA\#1, 5'GCAGGAUAUAAAGA A A C C U T T 3'; s i R A \# 2, 5' GCAUGGCA GAAUAUCUCUUTT3'; and siRNA\#3, 5' GGCUACUGACAAGAAUGAUTT3'. The construct that induced the strongest knockdown in PC-12 cells (shRNA\#3) was used for transfection. Cell transfection was performed using Lipofectamine 3000 (Invitrogen, CA, USA) according to the manufacturer's instructions. Briefly, $100 \mathrm{pmol} \mathrm{siFTH1}$ and $5 \mu$ of Lipofectamine reagent were diluted in $245 \mu \mathrm{l}$ OptiMEM (Life Technologies, CA, USA) for $5 \mathrm{~min}$ and then mixed gently. After incubation for $20 \mathrm{~min}$, the mixture was added to the cell cultures. A lentivirus overexpressing FTH1 was designed and synthesized by GenePharma. The final ratio of lentivirus to culture medium for transfection was 1:1000. After incubation for $24 \mathrm{~h}$, the culture medium was changed.

\section{Determination of Reduced Glutathione/Oxidized Glutathione Activity}

PC-12 cells $\left(5 \times 10^{3} /\right.$ well $)$ were cultured in 96 -well white plates. After treatment with $40 \mu \mathrm{M} 6-\mathrm{OHDA}$ for $24 \mathrm{~h}$, the cell culture medium was removed. The ratio of reduced glutathione (GSH)/oxidized glutathione (GSSG) was detected using the GSH/GSSG-Glo Assay (Promega, WI, USA). The cells were incubated with $50 \mu$ lof Total Glutathione Lysis Reagent or Oxidized Glutathione Lysis Reagent per well and shaken at room temperature for $5 \mathrm{~min}$. Then, $50 \mu \mathrm{l}$ of Luciferin Generation Reagent was added to each well, and the cells were incubated at room temperature for $30 \mathrm{~min}$. Then, a total of $100 \mu \mathrm{l}$ of Luciferin Detection Reagent was added to each well. The GSH/GSSG ratio was measured based on luminescence with a microplate reader (BioTek).

\section{Measurement of Lipid Peroxidation Generation by Flow Cytometry}

PC-12 cells $\left(1 \times 10^{5} /\right.$ well $)$ were seeded in 6 -well plates overnight and then treated with 6-OHDA for $24 \mathrm{~h}$. The cells were incubated with $10 \mu \mathrm{M} \mathrm{C11-BODIPY} \mathrm{(581/591)} \mathrm{(Life}$ Technologies) for $30 \mathrm{~min}$ at $37^{\circ} \mathrm{C}$, washed 3 times in PBS, and detached with trypsin (without EDTA, TransGen Biotech, Beijing, China). Fluorescence was measured by simultaneous acquisition of the green $(484 / 510 \mathrm{~nm})$ and red signals (581/ $610 \mathrm{~nm}$ ) using a CytoFLEX flow cytometer (Beckman Coulter, CA, USA). The ratio of the emission fluorescence intensities at 590 to $510 \mathrm{~nm}$ provided a readout of lipid peroxidation in cells. The data were analyzed by FlowJo v10.

\section{Detection of the Mitochondrial Membrane Potential by JC-1 Staining}

PC-12 cells $\left(1 \times 10^{5} /\right.$ well $)$ were cultured in 6 -well plates overnight. After incubation with 6-OHDA or other reagents, the cells were stained with JC-1 (Solarbio) following the manufacturer's protocol. Images were obtained using a fluorescence microscope (Olympus). Red fluorescence was generated in healthy mitochondria with a high membrane potential, whereas green fluorescence was produced in mitochondria with a decreased membrane potential. The change in mitochondrial membrane potential (MMP) was detected by measuring the change in green to red fluorescence. 


\section{Intracellular Ferrous lon Imaging by Fluorescence Microscopy}

$\mathrm{Fe}^{2+}$ distribution was assessed by a FeRhonox-1 staining kit (Goryo Chemical, Sapporo, Japan). PC-12 cells $\left(5 \times 10^{3} /\right.$ well) were seeded in a black 96-well microplate overnight. After the cells were incubated with 6-OHDA for $24 \mathrm{~h}$, the cell culture medium was removed, and the cells were rinsed twice with HBSS. Then, $1 \mu \mathrm{M}$ FeRhoNox-1 was added to the cell medium. Ferrous ion images were obtained using a fluorescence microscope (Olympus).

\section{Quantification of Iron Levels}

The Iron Assay Kit (Abcam) was used to quantify iron levels. PC-12 cells $\left(1 \times 10^{7}\right)$ were harvested for each assay after incubation with 6-OHDA for $24 \mathrm{~h}$. The cells were washed with cold PBS and then homogenized in 4 to 10 volumes of iron assay buffer with 10 to 15 passes or sonicated on ice. The samples were centrifuged at $16,000 \times g$ for $10 \mathrm{~min}$ to remove insoluble materials. A total of $5 \mu \mathrm{l}$ of assay buffer was added to each well to measure $\mathrm{Fe}^{2+}$ levels, and $5 \mu \mathrm{l}$ of iron reducer was added to each well to measure total iron levels. Then, the cells were incubated for $30 \mathrm{~min}$ at $25^{\circ} \mathrm{C}$, the iron probe was added, and the cells were incubated for $60 \mathrm{~min}$ at $25{ }^{\circ} \mathrm{C}$. Absorbance was measured at $593 \mathrm{~nm}$ by a microplate reader (BioTek).

\section{Determination of Autophagy by Confocal Microscopy}

PC-12 cells $\left(1 \times 10^{5} /\right.$ well $)$ were plated in a 6 -well plate. After incubation for $24 \mathrm{~h}$, the cells were infected with an adenovirus harboring tandem fluorescent mRFP-GFP-LC3 (Hanbio Inc., Shanghai, China) at a multiplicity of infection of 1000 . After the cells were incubated for another $24 \mathrm{~h}, 6-\mathrm{OHDA}(40 \mu \mathrm{M})$ was added, and the cells were incubated for an additional $24 \mathrm{~h}$. Autophagic flow was observed under a confocal microscope (Olympus).

\section{Observation of Mitochondrial Morphology by Transmission Electron Microscopy}

PC-12 cells $\left(1 \times 10^{6} /\right.$ well $)$ were harvested by centrifugation at $1000 \mathrm{rpm}$ for $2 \mathrm{~min}$. The cells were fixed with $2.5 \%$ glutaraldehyde at $4{ }^{\circ} \mathrm{C}$ for $2 \mathrm{~h}$ and washed 6 times with $0.1 \mathrm{M}$ phosphate buffer. Then, the cells were fixed with $1 \%$ osmium acid for $2 \mathrm{~h}$ and washed 3 times with $0.1 \mathrm{M}$ phosphate buffer. After that, the cells were fixed with $1 \%$ osmium tetroxide and dehydrated through an alcohol gradient before being embedded in resin. The samples were detected using transmission electron microscopy (JEM-1400 PLUS, Tokyo, Japan) after double staining with uranium acetate and lead citrate.

\section{Statistical Analysis}

All statistical tests were performed using Student's $t$ test, and the data were analyzed with GraphPad Prism 5.0 (GraphPad Software, San Diego, CA) or SPSS (version 17.0; SPSS, Chicago, IL). The data were considered statistically significant when $* p<0.05, * * p<0.01$, or $* * * p<0.001$.

\section{Result}

\section{Differential mRNA Expression Profile of PD Model Rats}

To identify differentially expressed mRNAs between tissues from PD model rats and tissues from normal control rats, we used the BGIseq500 platform to analyze differences in gene expression. A total of 23,111 mRNAs were expressed in the normal and model groups (Fig. 1A). A volcano plot and heatmap showed that the mRNA expression patterns in the 2 groups were significantly different. A total of 597 differentially expressed genes were identified, namely 404 upregulated and 193 downregulated mRNAs $(p<0.01, \log 2$-fold fold change $>2$, Fig. 1B, C). To determine the biological effects of the significantly differentially expressed genes, we performed gene ontology (GO) analysis and Kyoto Encyclopedia Gene and Genomes (KEGG) pathway analysis to determine the important pathways associated with the differentially expressed genes. The KEGG pathways of neurodegenerative diseases, nervous system, and aging were selected to construct a network (Supplementary Fig. 1A, B). Based on the network, 148 closely linked genes were enriched, and these genes were subjected to KEGG pathway analysis. Among the enriched pathways, ferroptosis was closely related to the pathogenesis of PD. FTH1 was involved in the ferroptosis pathway, and it was downregulated in the PD model group compared with the control group (Fig. 1D, E).

\section{FTH1 Was Downregulated and Related to Development in the 6-OHDA Model of PD}

HE staining was used to observe morphological changes in the nerve cells in a rat model of PD. Compared with those in the normal and sham groups, the number of nerve cells in the 6OHDA model group was reduced, and the cells were sparse and irregularly arranged (Fig. 2A). $\alpha$-Syn is closely related to the pathogenesis of $\mathrm{PD}$ and causes neuronal cell death. TH is a protein marker of dopaminergic neurons in the nervous system $[1,2]$. The immunohistochemistry and Western blot results showed that the expression of $\alpha$-syn was increased and that the expression of TH and FTH1 were decreased (Fig. 2B, $\mathrm{C}$ ) in the model group compared with the normal and sham groups. PC-12 cells were incubated with or without 6-OHDA 
Fig. 1 Differential gene expression of mRNA. (A) Venn diagram of differentially expressed genes in the normal group and the model group. (B, C) Volcano plot and heatmap of differentially expressed mRNAs. (D) The fragments per kilobase of transcript per million fragments mapped (FPKM) of FTH1 in the PD rat model. (E) The significantly enriched KEGG pathways of the differentially expressed mRNAs
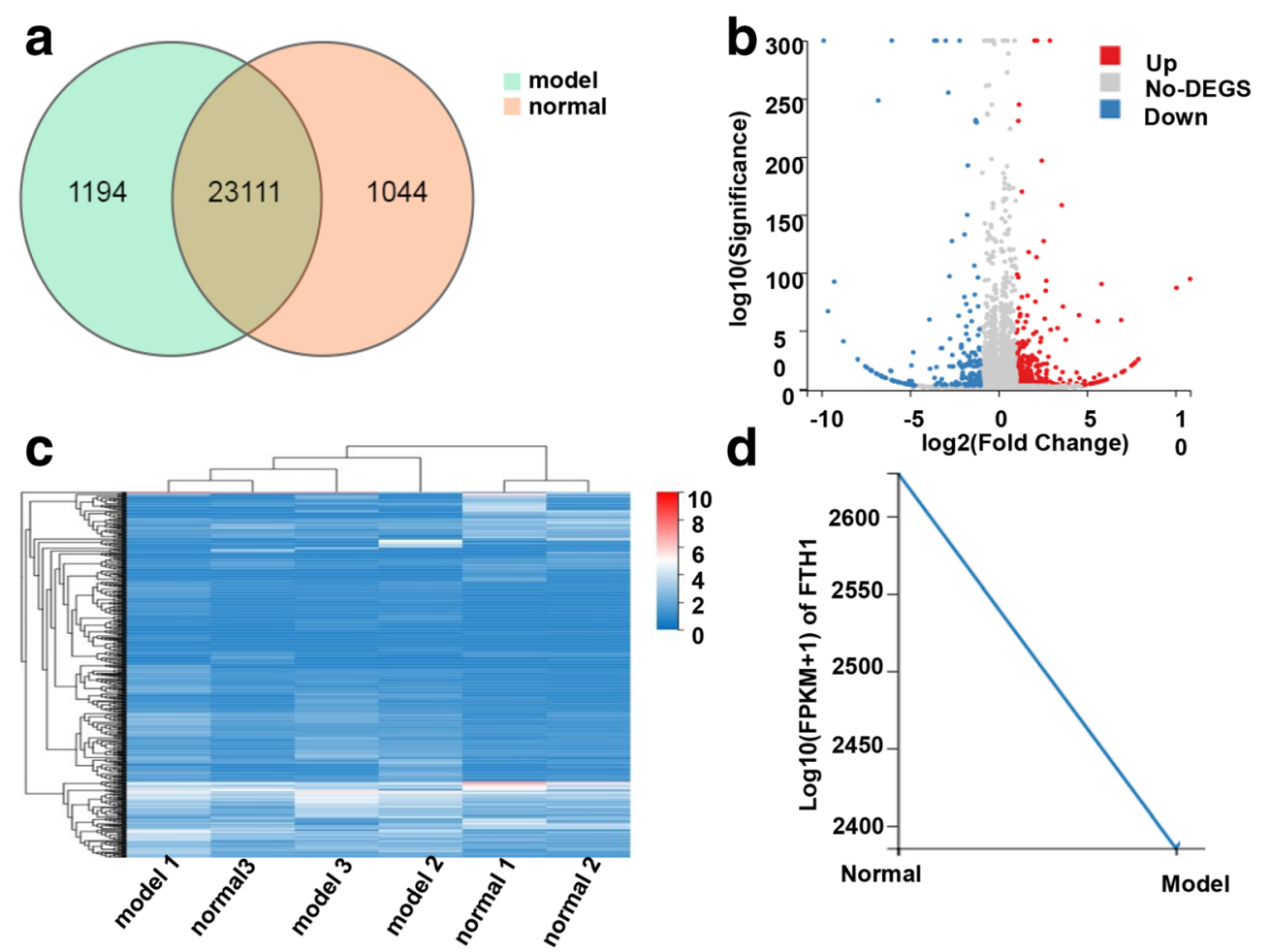

e

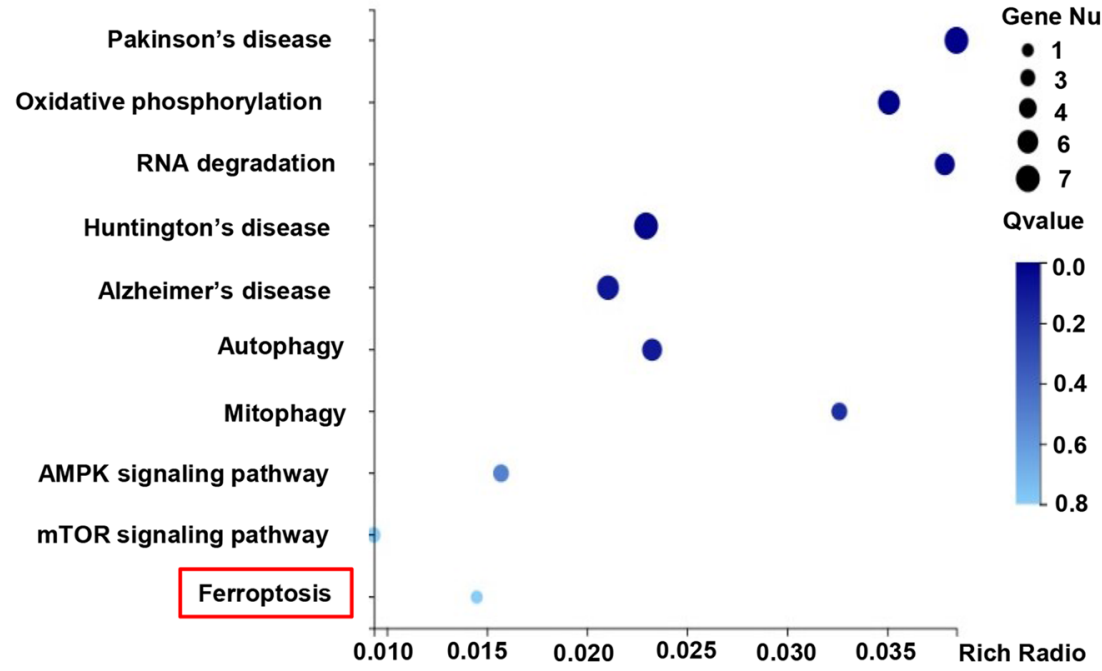

for 24 h. 6-OHDA exhibited significant cytotoxic activity at a concentration of 25.0 to $100.0 \mu \mathrm{M}$, and the $\mathrm{IC}_{50}$ value was $49.3 \pm 3.4 \mu \mathrm{M}$ (Fig. 2D). Live/dead staining indicated that compared with control treatment, treatment of PC-12 cells with 6-OHDA at a concentration 25.0 to $100.0 \mu \mathrm{M}$ resulted in a lower density of live cells (green) and a higher density of dead cells (red) (Fig. 3). Then, we examined the effect of treatment with $40 \mu \mathrm{M} 6-\mathrm{OHDA}$ for 12,24 , or $48 \mathrm{~h}$ on the viability of PC-12 cells. The results showed that 6-OHDA had no apparent effect on cell activity after treatment for $12 \mathrm{~h}$ but decreased cell viability by $86.8 \%$ after treatment for $48 \mathrm{~h}$. Considering that live cells were required for subsequent experiments, we chose $40 \mu \mathrm{M} 6-$ OHDA and $24 \mathrm{~h}$ as the appropriate concentration and treatment period for further experiments (Fig. 2E). Our results also revealed that $\mathrm{TH}$ and FTH1 were downregulated in PC-12 cells treated with 6OHDA compared with control-treated cells, whereas the expression of $\alpha$-syn was increased significantly (Fig. 2F). Taken together, these results confirmed that the expression of FTH1 was downregulated in the PD model group compared with the control group, suggesting that FTH1 is associated with the development of this model.

\section{FTH1 Exerted Cytoprotective Activity and Regulated Protein Expression in PC-12 Cells}

To determine the regulatory role of FTH1, we next investigated the cytoprotective effect of FTH1 and its effect on protein 
a

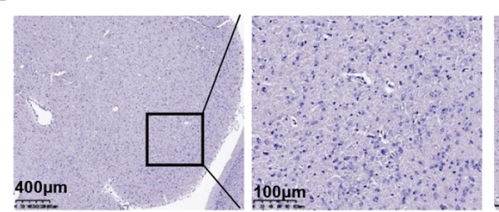

b

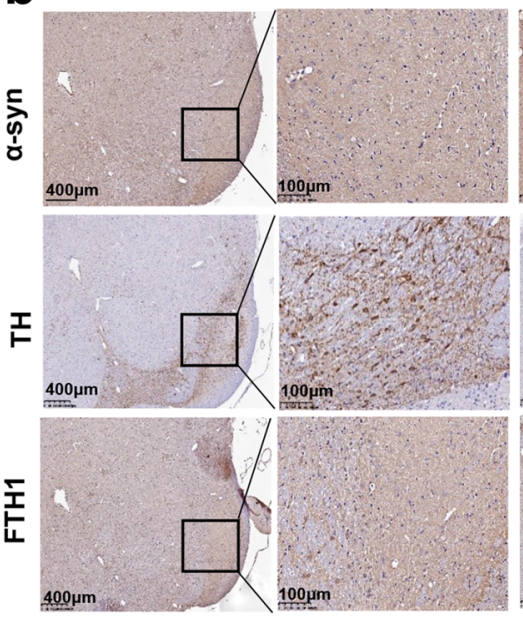

C

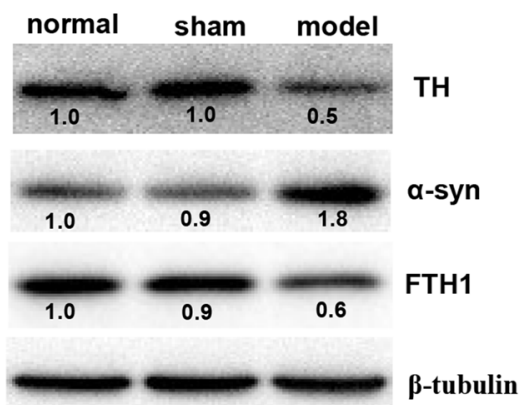

e

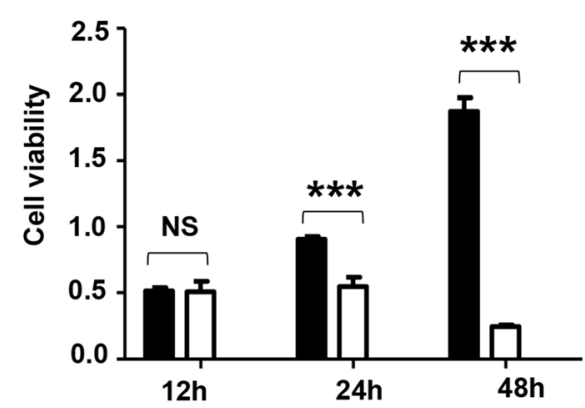

Fig. 2 FTH1 was downregulated in the 6-OHDA model of PD. Morphological changes in nerve cells and protein expression were analyzed in the substantia nigra (SN) tissue from the normal, sham, and model groups. (A) HE staining (magnification $\times 4$ and $\times 20$ ). (B) Immunohistochemical staining of $\alpha$-syn, TH, and FTH1 (magnification $\times 4$ and $\times 20$ ). (C) Western blot analysis of the expression of TH, $\alpha$-syn, and FTH1. The data were normalized to the control group values. (D) PC12 cells were untreated or treated with $6.3,12.5,25.0,50.0$, or $100.0 \mu \mathrm{M}$ sham

model
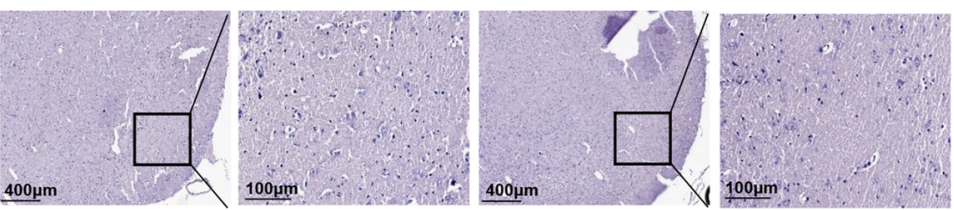

sham

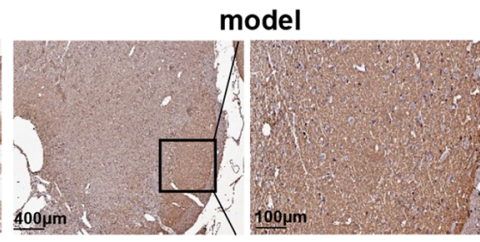

$1004 \mathrm{~m}$
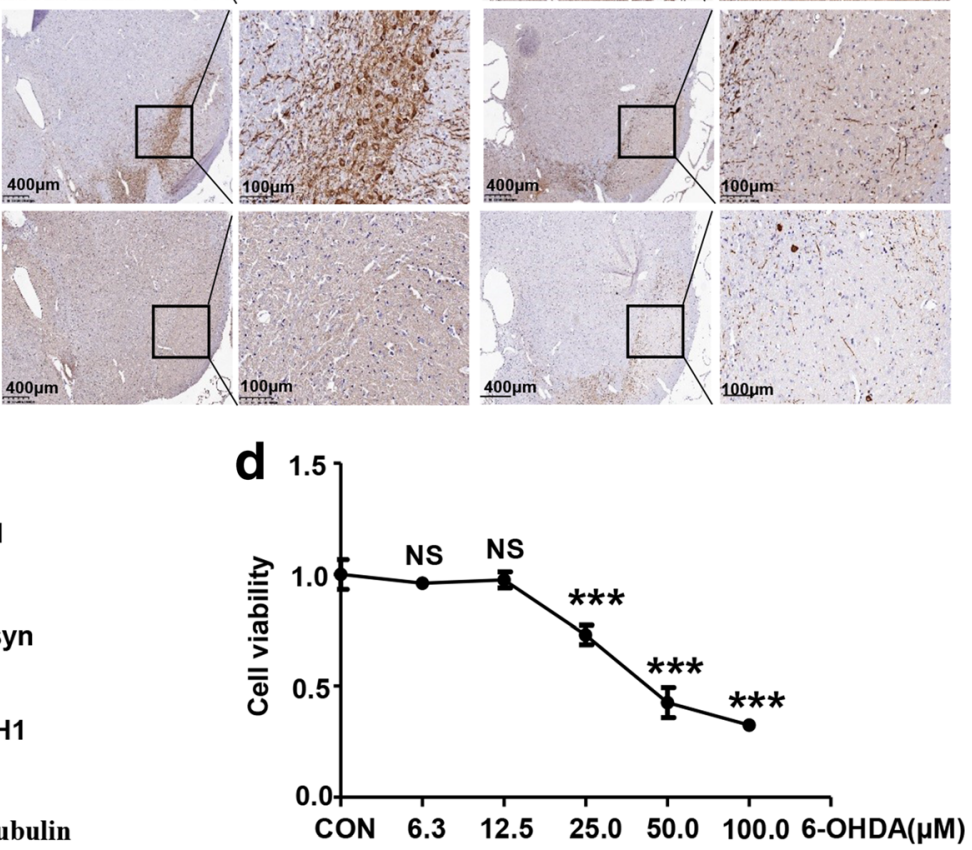

$\mathbf{f}$

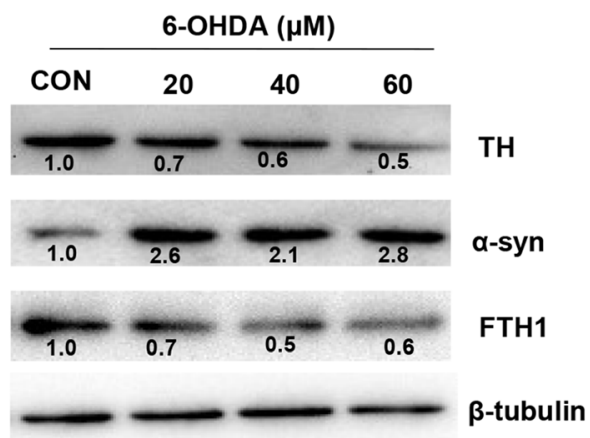

6-OHDA for $24 \mathrm{~h}$, and cell viability was evaluated by the MTS assay. (E) PC-12 cells were untreated or treated with $40 \mu \mathrm{M} 6-O H D A$ for 12,24 , or $48 \mathrm{~h}$, and cell viability was evaluated by the MTS assay. (F) PC-12 cells were untreated or treated with 20,40 , or $60 \mu \mathrm{M}$ 6-OHDA for $24 \mathrm{~h}$. The protein expression of TH, $\alpha$-syn, and FTH1 was determined by Western blotting. The data are expressed as means \pm S.D.; $n \geq 3$ per group for all the studies $\left(t\right.$ test, $\left.* * p<0.01,{ }^{* * *} p<0.001\right)$. The data were normalized to the control group values 
Fig. 3 Fluorescence detection of living and dead cells. PC-12 cells were treated with $6.3,12.5,25.0$, 50.0 , or $100.0 \mu \mathrm{M} 6-\mathrm{OHDA}$ for $24 \mathrm{~h}$. PI was used to detect dead cells (red), and calcein-AM was used to detect living cells (green). The cells were imaged under a fluorescence microscope
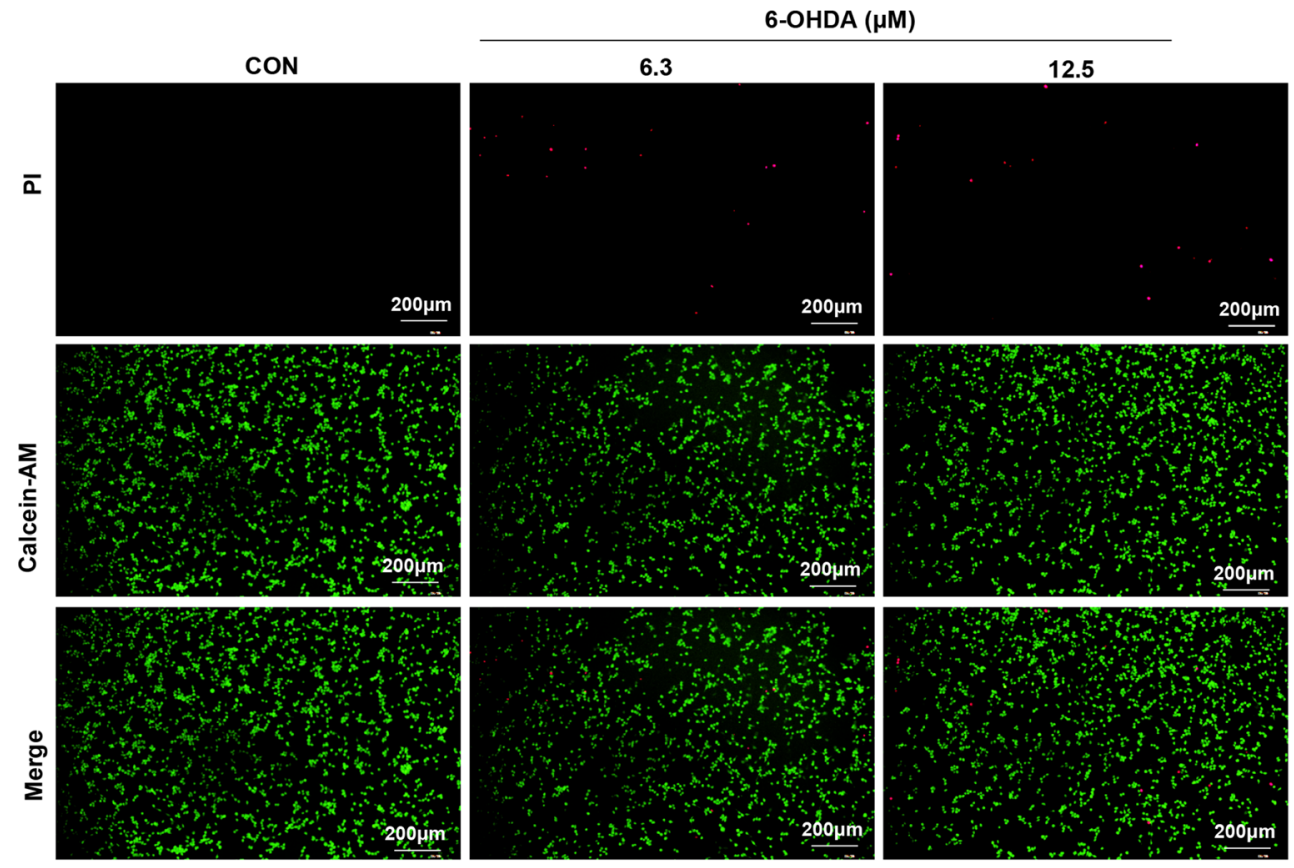

50
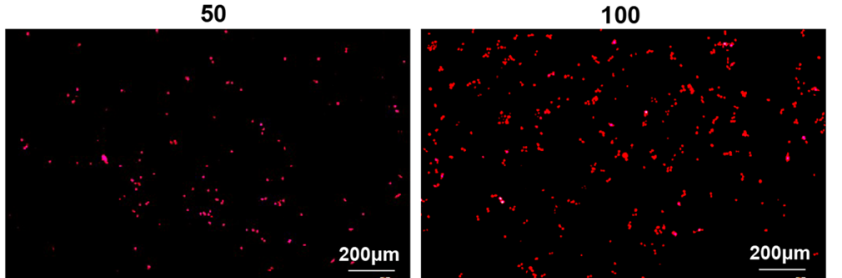

$\bar{a}$
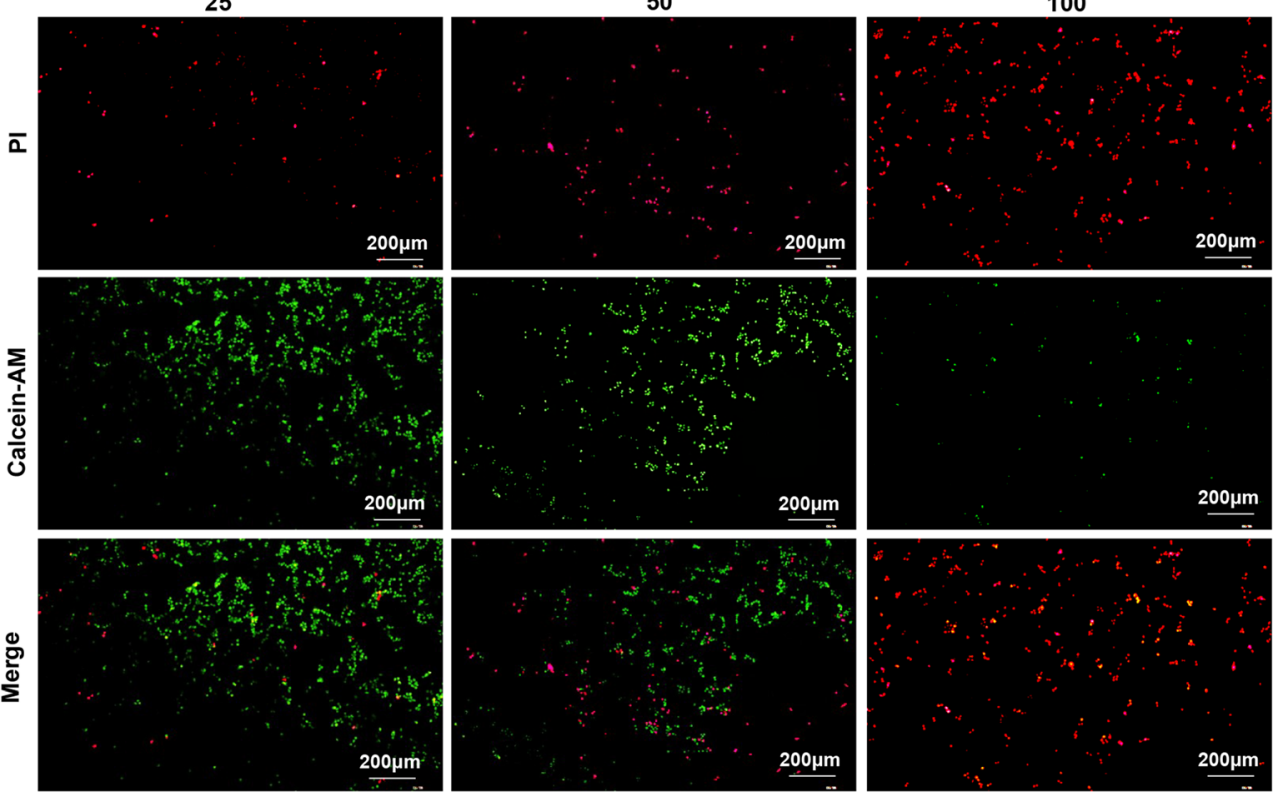

expression in the cell model of PD. PC-12 cells were transfected with FTH1 siRNA or lentivirus to knock down or increase the expression of FTH1, respectively (Fig. 4A, B). The results showed that FTH1 knockdown in PC-12 cells led to decreased cell viability (Fig. 4C), whereas FTH1 overexpression resulted in increased viability of PC-12 cells (Fig. 4D). We next studied the effect of FTH1 overexpression or knockdown on the viability of cells incubated with 6-OHDA. Our results showed that the viability of FTH1 knockdown cells treated with $40 \mu \mathrm{M} 6-\mathrm{OHDA}$ was significantly decreased compared with that of 6-OHDA-treated cells (Fig. 4C). In contrast, overexpression of FTH1 mitigated the effect of 6-
OHDA on cell viability. The cell viability rate decreased by $40.8 \%$ in the vector-treated group upon treatment with 6OHDA, whereas the cell viability rate decreased by $14.4 \%$ in FTH1-overexpresing cells upon treatment with 6-OHDA (Fig. 4D). The regulatory effect of FTH1 on PD-related protein expression was assessed by Western blotting. As shown in Fig. 4E and F, FTH1 knockdown decreased the expression of TH, especially in PC-12 cells treated with 6-OHDA. FTH1 overexpression showed the opposite effect, indicating that FTH1 exerted cytoprotective activity and regulated protein expression in the 6-OHDA model of PD. The results demonstrated that FTH1 is involved in the pathogenesis of PD. 
Fig. 4 FTH1 mediated cytoprotective activity and altered protein expression in the cell model of PD. PC-12 cells were treated with 6-OHDA $(40 \mu \mathrm{M})$ for $24 \mathrm{~h}$ to establish a cell model of PD. (A) PC-12 cells were transfected with FTH1 siRNA or siRNA control (NC). (B) PC-12 cells were infected with FTH1 overexpression lentivirus or control vector lentivirus (VEC). The expression of FTH1 was analyzed using Western blotting. The effect of FTH1 knockdown (C) or overexpression (D) on cell viability was determined using the MTS assay. The expression of TH in FTH1 knockdown (E) or FTH1-overexpressing (F) PC-12 cells was analyzed using Western blotting ( $n \geq 3$ assays, $t$ test, $* p<0.05, * * p<0.01)$. The data were normalized to the control group values a
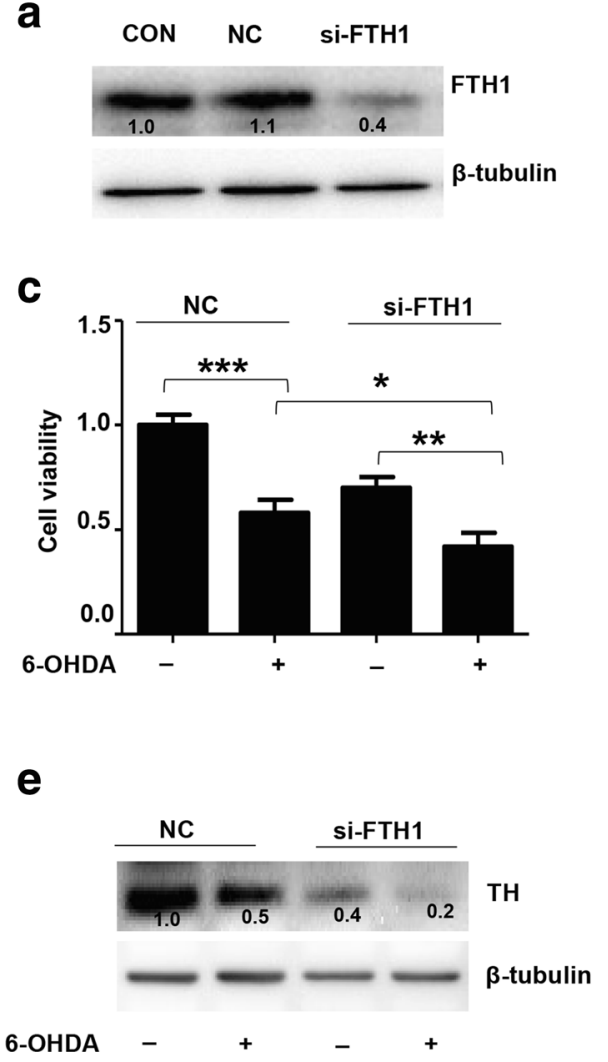

b

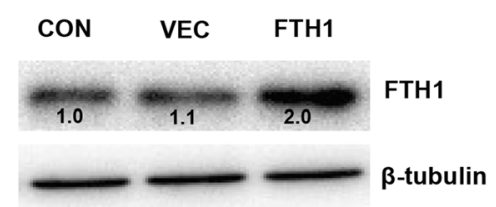

d

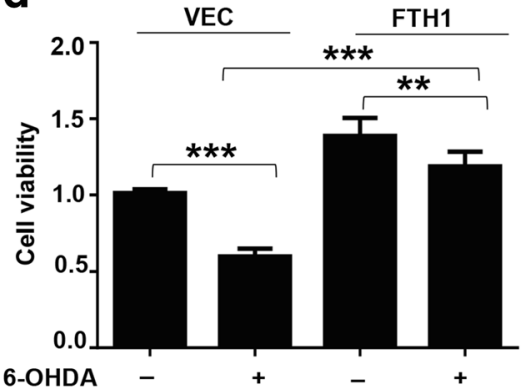

f

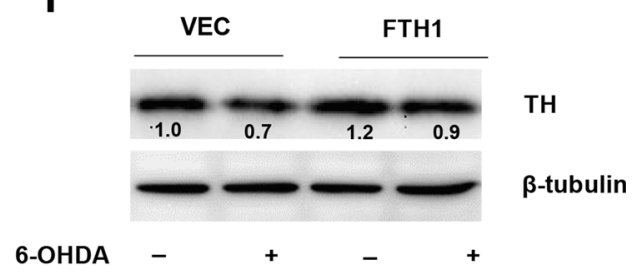

\section{Induction of Ferroptosis in the 6-OHDA Model of PD}

FTH1 has been shown to be involved in ferroptosis. Ferroptosis is an iron-dependent type of neuronal cell death and has been observed in recent studies on PD. To investigate 6-OHDA-induced cell death through the ferroptosis pathway, we measured the level of $\mathrm{Fe}^{2+}$, the expression of GPX4, lipid peroxidation, GSH levels, and the MMP as indicators of ferroptosis. Iron is a necessary mediator of ferroptosis. As shown in Fig. 5A and B, the level of total iron was increased by $23.3 \%$, and that of $\mathrm{Fe}^{2+}$ was increased by $36.8 \%$ in PC-12 cells treated with 6-OHDA compared with control cells. In addition, the results of iron staining confirmed that $\mathrm{Fe}^{2+}$ accumulated in PC-12 cells (Fig. 5C). Moreover, the expression of GPX4 was significantly decreased in the cell model group of PD compared with the control group (Fig. 5D, E). GSH depletion leads to an increase in lipid peroxides and iron for ferroptosis. As shown in Fig. 5F, 6-OHDA decreased the GSH/GSSG ratio in PC-12 cells. The level of lipid peroxides was increased by $42.4 \%$ in 6-ODHA-treated PC-12 cells compared with control cells (Fig. 5G, H). Mitochondrial damage is also required for ferroptosis in neuronal cells [24, 25]. Further detection of mitochondrial dysfunction was performed by JC1 staining. The results indicated that the MMP $(\Delta \psi \mathrm{m})$ was significantly decreased after 6-OHDA treatment, as evidenced by an increased ratio of green to red fluorescence (Fig. 5I).

\section{The Effect of a Ferroptosis Inducer or Inhibitor on PC-} 12 Cells

All the above results showed that ferroptosis was induced by 6-OHDA, and we next studied the effect of inducer or inhibitor of ferroptosis in PC-12 cells. The effect of the active ferroptosis inducer erastin [13] on cell viability and the expression of FTH1 were investigated in ferroptotic PC-12 cells. As shown in Fig. 6A, erastin treatment enhanced the effect of 6-OHDA. The cell viability rate of $\mathrm{PC}-12$ cells treated with $40 \mu \mathrm{M} 6$-OHDA alone was $60.8 \%$, whereas the cell viability rate was decreased to $46.4 \%$ in cells treated with the combination of erastin and 6-OHDA. The expression of GPX4, TH, and FTH1 was significantly reduced in cells treated with the combination of erastin and 6-OHDA compared with those treated with 6-OHDA alone (Fig. 6B). All of these effects were reduced by the iron chelator DFO and the ferroptosis inhibitor Fer-1 $[13,26]$. The cell viability rate increased by $20.4 \%$ and $32.7 \%$ in cells treated with the combination of DFO and 6-OHDA and cells treated with the combination of Fer-1 and 6-OHDA, respectively (Fig. 6C, E). The expression of GPX4, TH, and FTH1 was also increased in cells treated with ferroptosis inhibitors compared with those treated with 6OHDA alone (Fig. 6D, F). Overall, these results further suggested that 6-OHDA induced some degree of ferroptotic death and that FTH1 was degraded during ferroptosis in PC-12 cells. 
a

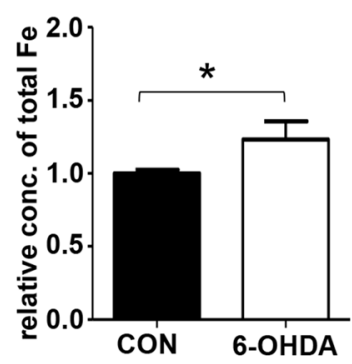

d

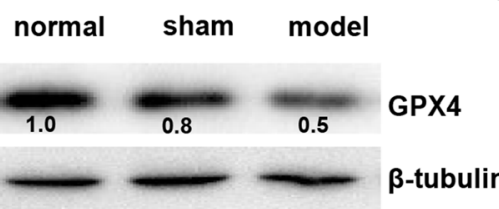

g

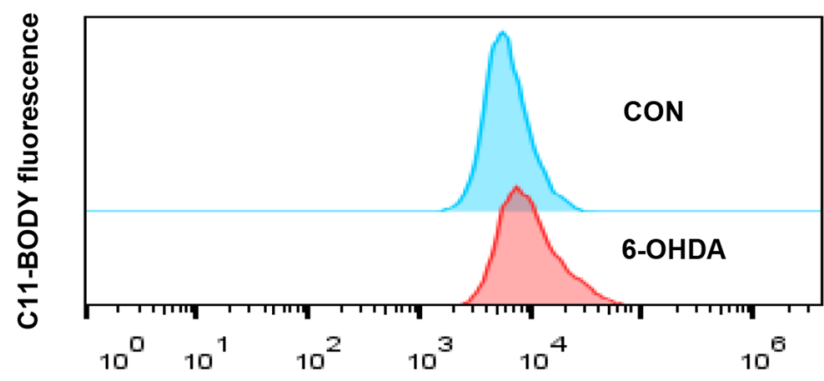

i
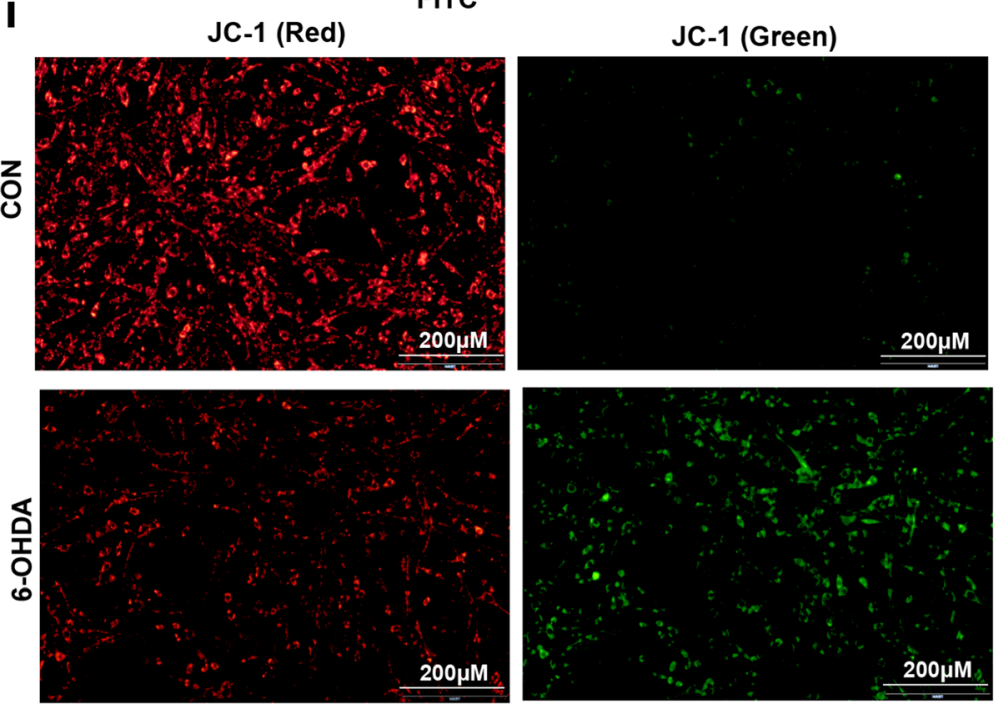

Fig. 5 Induction of ferroptosis in the 6-OHDA model of PD. (A, B) PC12 cells were treated with $40 \mu \mathrm{M}$ 6-OHDA for $24 \mathrm{~h}$. The levels of total iron and $\mathrm{Fe}^{2+}$ were measured by the Iron Assay Kit. (C) Iron staining (red) was performed using a FeRhoNox-1 fluorescent imaging probe as described in the "Materials and Methods" section. The expression of GPX4 in rat SN tissue (D) and PC-12 cells (E) treated with 6-OHDA was analyzed by Western blotting. The data were normalized to the
C CON 6-OHDA

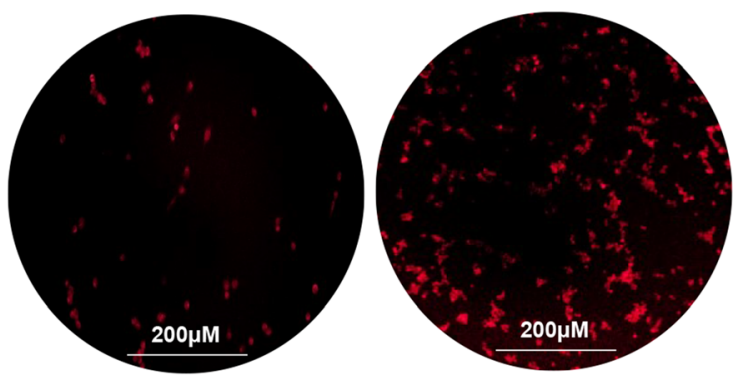

f

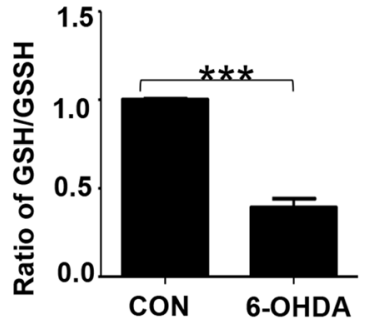

h

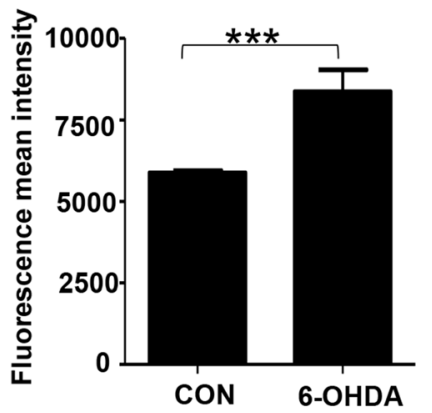

Merge
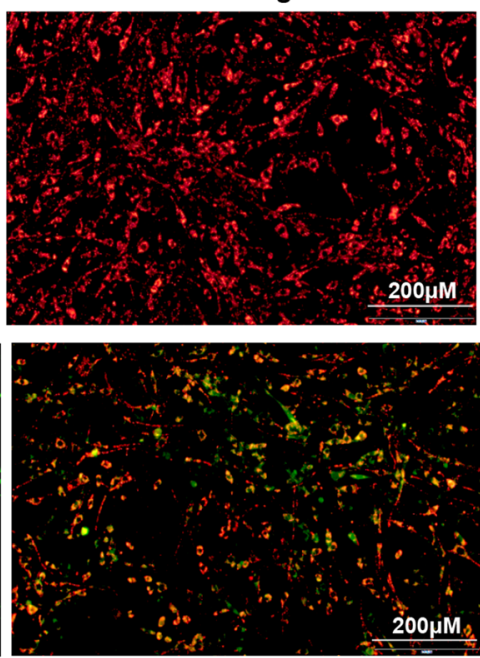

control group values. (F) The ratio of GSH/GSSG in PC-12 cells was detected using the GSH/GSSG-Glo Assay (Promega). (G) Fluorescent BODIPY staining and FACS analysis $(\mathrm{H})$ were used to evaluate the formation of lipid peroxides in PC-12 cells. (I) JC-1 staining was employed to assess mitochondrial damage by measuring the ratio of green to red fluorescence as described in the "Materials and Methods" section ( $n \geq 3$ assays, $t$ test, $* p<0.05, * * * p<0.001$ ) 
Fig. 6 The effect of an inducer or inhibitor of ferroptosis on PC-12 cells. PC-12 cells were treated with $40 \mu \mathrm{M}$ 6-OHDA in combination with an inducer $(1 \mu \mathrm{M}$ erastin) or inhibitor ( $25 \mu \mathrm{M}$ DFO and $2.5 \mu \mathrm{M}$ Fer-1) of ferroptosis for $24 \mathrm{~h}$. Cell viability was determined by the MTS assay (A, C, and E). The expression of GPX4, FTH1, and TH was analyzed using Western blotting (B, D, and F) $(n \geq 3$ assays, $t$ test, $* p<0.05$, $* * p<\overline{0} .01, * * * p<0.001)$. The data were normalized to the control group values
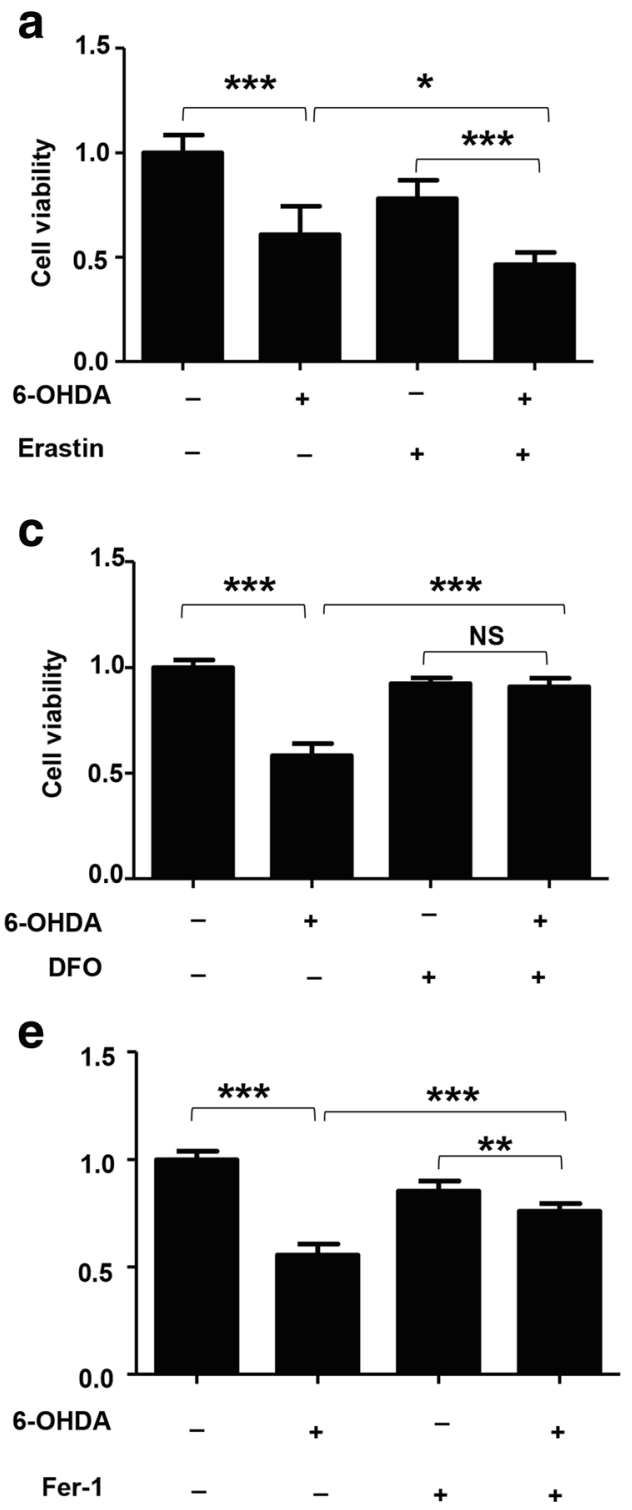

b
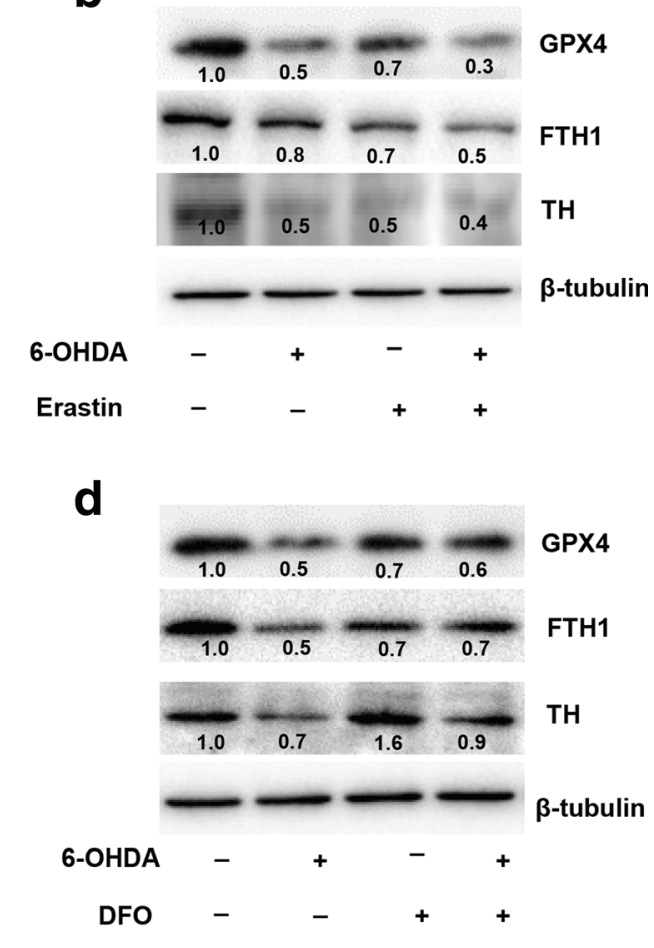

f

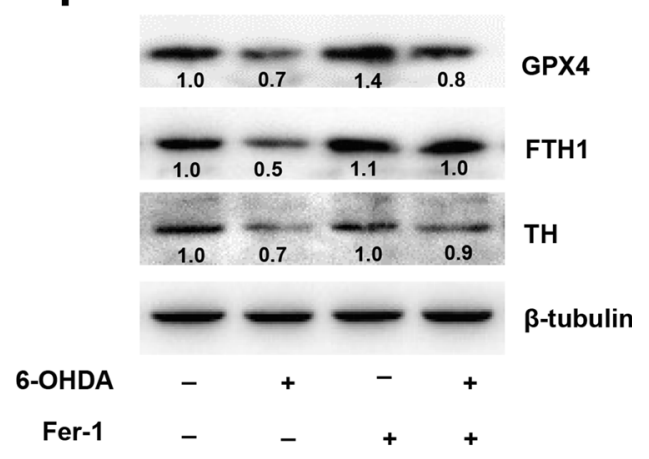

\section{FTH1 Mediated Ferroptosis in PC-12 Cells}

Next, we checked whether 6-OHDA-induced cell ferroptosis was regulated by FTH1. The results were examined in cells treated with si-FTH1 or an FTH1 overexpression lentivirus. As shown in Fig. 7A and B, the expression of GPX4 was reduced in FTH1 knockdown cells treated with 6-OHDA compared with control cells treated with 6-OHDA, but increased in cells overexpressing FTH1 and treated with 6OHDA compared with control cells treated with 6-OHDA. Ferrous ion imaging also showed that the level of $\mathrm{Fe}^{2+}$ was increased by si-FTH1 (Fig. 7C). Overexpression of FTH1 mitigated the effect of 6-OHDA on the upregulation of $\mathrm{Fe}^{2+}$ levels (Fig. 7D). JC-1 staining indicated that the MMP was increased by FTH1 knockdown and decreased by FTH1 overexpression. Knockdown of FTH1 enhanced the effect of 6OHDA-induced mitochondrial dysfunction; in contrast, overexpression of FTH1 mitigated the effect of 6-OHDA (Fig. 8A, B). These data provided further evidence that 6OHDA-induced ferroptosis was mediated by FTH1 and that overexpression of FTH1 mitigated the effect of ferroptosis in the cell model of PD.

\section{FTH1 Induced Ferroptosis Through Ferritinophagy in the 6-OHDA Model of PD}

The above results confirmed the role of FTH1 in ferroptosis in the 6-OHDA model of PD. FTH1 is also a major player in ferritinophagy, so we next studied the regulatory mechanism of FTH1 in ferritinophagy. As shown in Fig. 9A and B, the expression of NCOA4 and the ratio of LC3B-II/LC3B-I were significantly increased in the $\mathrm{PD}$ group compared with the control group. PC-12 cells were infected with a GFP-RFPLC3 adenovirus and then treated with $40 \mu \mathrm{M}$ 6-OHDA for 
Fig. 7 FTH1 mediated ferroptosis in PC-12 cells. The expression of GPX4 and FTH1 was evaluated in PC-12 cells treated with the knockdown (A) or overexpression (B) vector combined with $40 \mu \mathrm{M} 6$ 6-OHDA. FTH1 expression was analyzed using Western blotting. The data were normalized to the control group values. A FeRhoNox-1 fluorescent imaging probe was employed for ferrous ion imaging of PC-12 cells treated with the knockdown (C) or overexpression (D) vector combined with $40 \mu \mathrm{M}$ 6-OHDA. All data are representative of at least 3 independent experiments
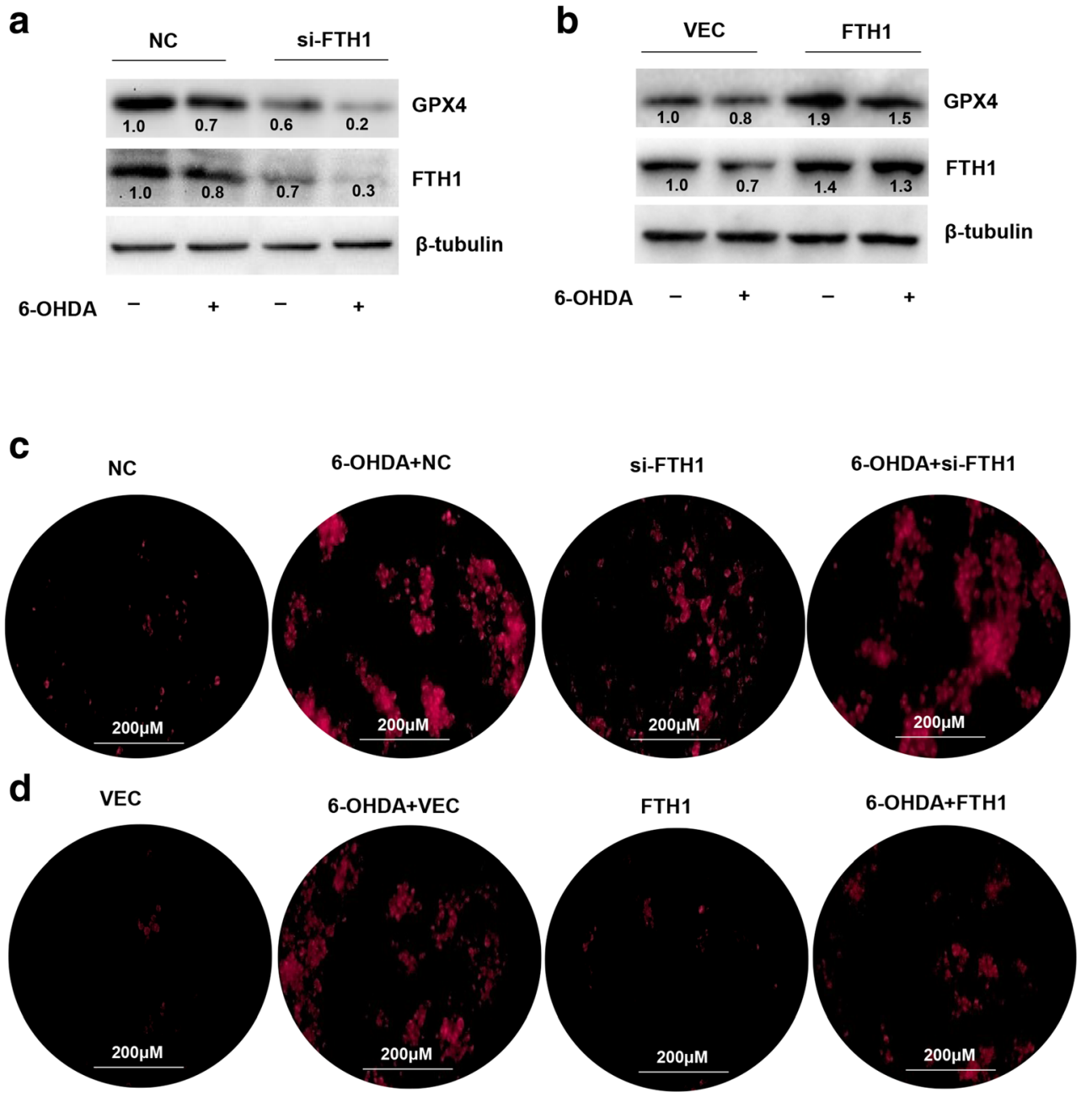

$24 \mathrm{~h}$, and autophagic flux was monitored. As GFP is sensitive to $\mathrm{pH}$, the decrease in the $\mathrm{pH}$ following the fusion of lysosomes to autophagosomes quenches GFP fluorescence. Thus, autophagosomes appear yellow due to the combination of red and green fluorescence, whereas autolysosomes appear red. As shown in Fig. 9C, merged red and green puncta appeared as yellow puncta in PC-12 cells, indicating that autophagic flux was successfully induced. In addition to increased accumulation of LC3, more red puncta were observed in cells treated with 6-OHDA $(40 \mu \mathrm{M})$ than in control cells, suggesting that the number of autophagosomes and autophagic flux were increased (Fig. 9C). These results demonstrated that ferritinophagy was induced in PC-12 cells treated with 6OHDA. To further investigate the role of FTH1 in ferritinophagy in the cell model of PD, PC-12 cells were transfected with si-FTH1 or an overexpression lentivirus. The results showed that the expression of NCOA4 and the ratio of LC3-II/LC3-I were increased when FTH1 was knocked down, especially in cells treated with 6-OHDA, whereas FTH1 overexpression mitigated the effect of 6OHDA on the expression of NCOA4 and the ratio of LC3II/LC3-I (Fig. 10A, B). The defining feature of ferritinophagy is mitochondrial iron overload with subsequent mitochondrial damage. The effects of FTH1 on mitochondrial morphology were studied using electron microscopy. As shown in Fig. 11A, in PC-12 cells treated with 6-OHDA or si-FTH1, mitochondrial cristae were broken, and the mitochondrial structure was fuzzy. In cells treated with the combination of si-FTH1 and 6-ODHA, the mitochondria displayed apparent swelling and vacuolization, whereas FTH1 overexpression alleviated mitochondrial impairment during ferritinophagy (Fig. 11B).

Previous studies showed that CQ and Baf-A1, as inhibitors of autophagy, decrease autophagosome-lysosome fusion [27]. We studied the effects of CQ or Baf-A1 on cell viability in the presence or absence of si-FTH1 in PC-12 cells treated with 6-OHDA. As shown in Fig. 10C, the cell viability was $55.9 \%$ in cells treated with 6-OHDA only, whereas the viability rate increased to $73.7 \%$ and $61.3 \%$ in cells treated with the combination of CQ and 6-OHDA or the combination of BafA1 and 6-OHDA. Additionally, the viability rate decreased to $63.0 \%$ and $49.2 \%$ in FTH1 knockdown cells treated with the combination of CQ and 6-OHDA or the combination of BafA1 and 6-OHDA. CQ and Baf-A1 mitigated the effect of 6- 
Fig. 8 FTH1 inhibited mitochondrial dysfunction in PC12 cells. The MMP in PC-12 cells treated with the knockdown (A) or overexpression (B) vector combined with $40 \mu \mathrm{M} 6-\mathrm{OHDA}$ was determined by JC-1 staining ( $n \geq 3$ assays)

(a)

\section{2 NC}
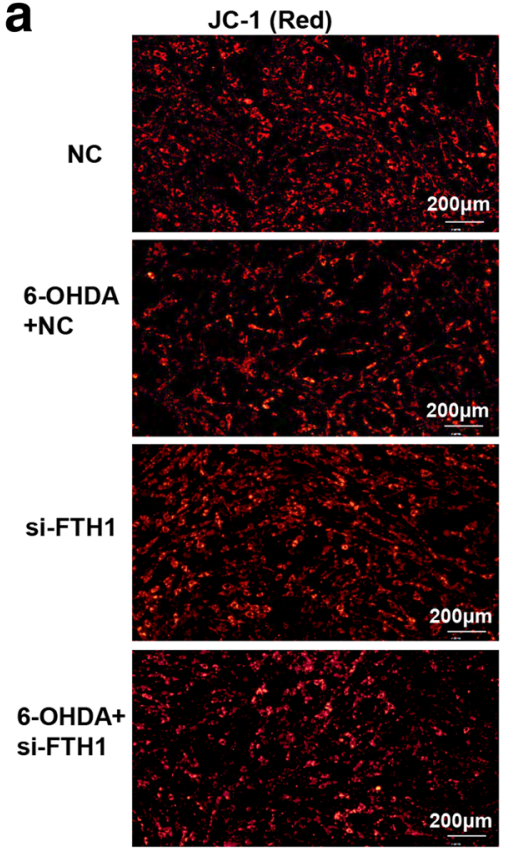

b
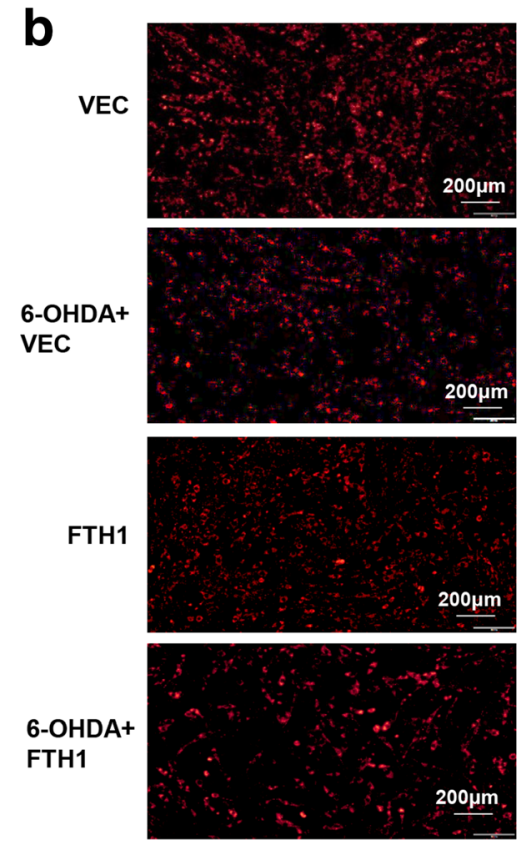
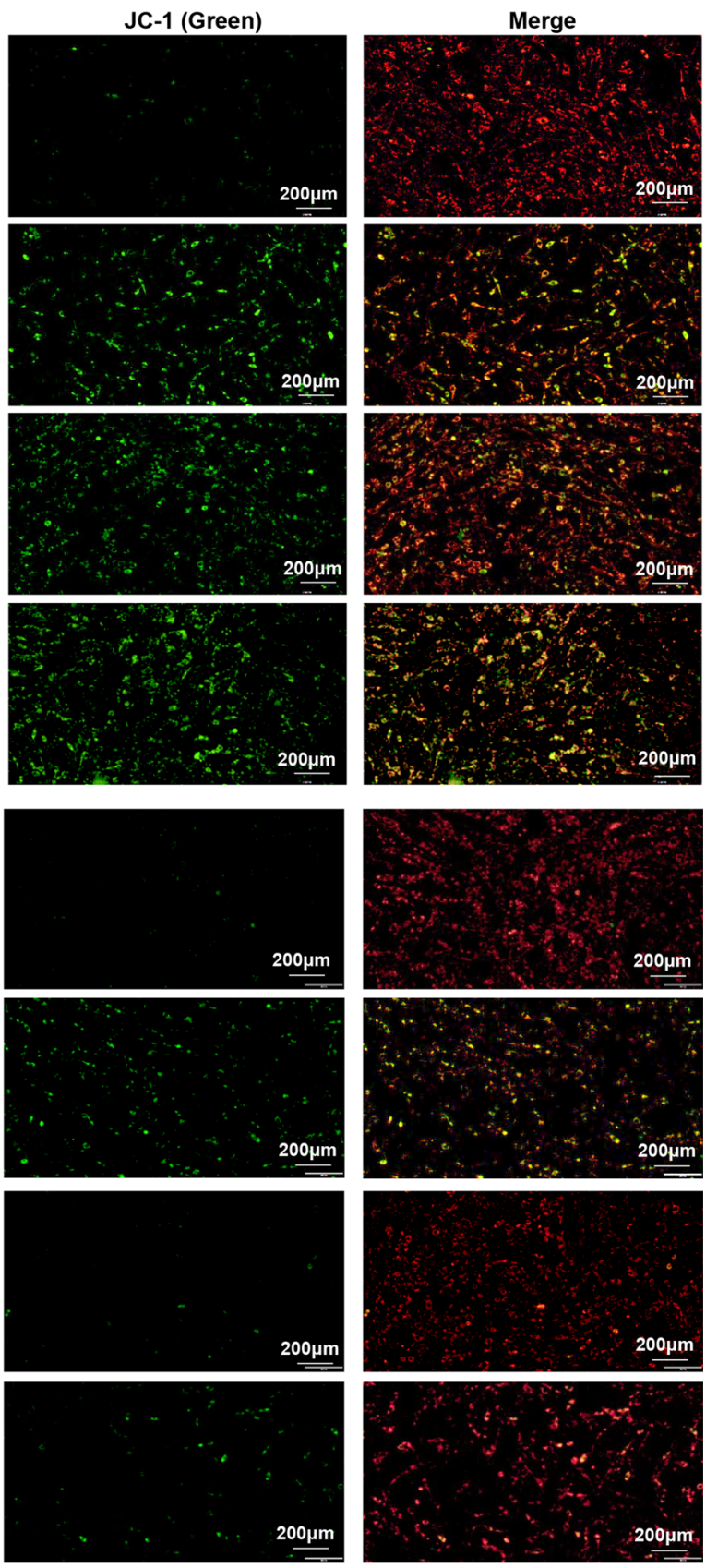

OHDA on the expression of GPX4, FTH1, NCOA4, and TH. si-FTH1 partly inhibited the effect of CQ and that of Baf-A1 in PC-12 cells (Fig. 10D). These results showed that FTH1 regulated cell viability and the expression of related proteins by regulating the ferritinophagy process. Inhibition of ferritinophagy decreased the expression of FTH1 and limited the effect of FTH1, but a low level of FTH1 was the major component that affected the entire pathway.

Thus, we speculated that FTH1 was degraded by ferritinophagy to provide a low level of FTH1 as a signal for the release of iron, causing ferroptosis. The regulation of FTH1 and ferritinophagy might be cyclic, as shown in Fig. 12. At a low level, FTH1 recruited LC3 and selectively bound to NCOA4 in autophagosomes, and then ferritinophagy was induced to degrade FTH1. After FTH1 is downregulated, it further promotes ferritinophagy. Eventually, FTH1 is maintained at an extremely low level, resulting in the accumulation of free ferrous ions and mitochondrial impairment, eventually leading to cell death due to ferroptosis in the 6-OHDA model of PD. The findings indicate that there is a positive relationship between ferritinophagy and ferroptosis and that FTH1 is a key link between these processes in the 6-OHDA model of PD. 
Fig. 9 Induction of

ferritinophagy in the 6-OHDA model of PD. The expression of NCOA4, LC3B-I, and LC3B-II in $\mathrm{PC}-12$ cells $(\mathrm{A})$ and $\mathrm{SN}$ tissue (B) from rats was determined by Western blotting. The data were normalized to the control group values. (C) PC-12 cells were infected with a GFP-RFP-LC3 adenovirus for $24 \mathrm{~h}$ and then treated with $40 \mu \mathrm{M}$ 6-OHDA for $24 \mathrm{~h}$.

Autophagosomes, which are represented by yellow puncta in the merged images, and

autolysosomes, which are represented by red puncta, were detected with fluorescence microscopy. The results represent the means of 3 independent experiments a

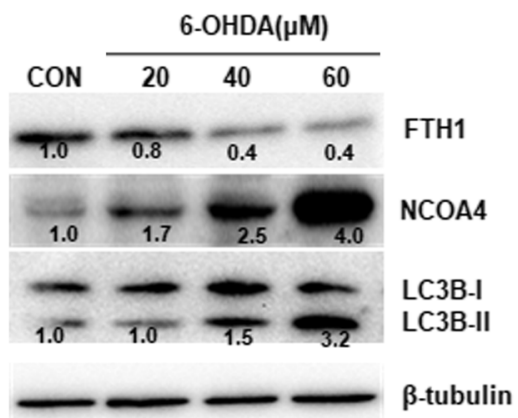

b

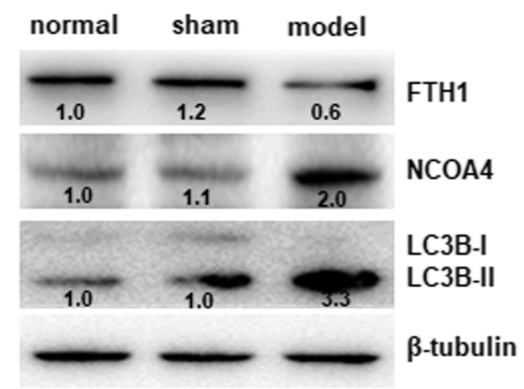

C CON

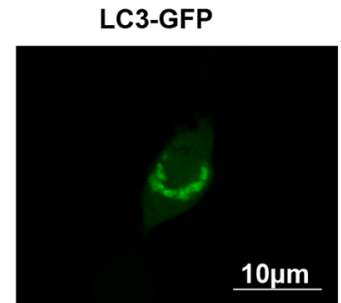

6-OHDA

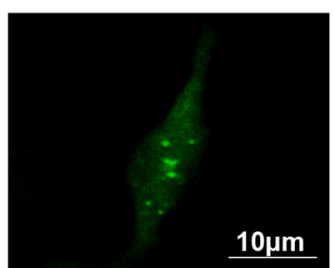

LC3-RFP
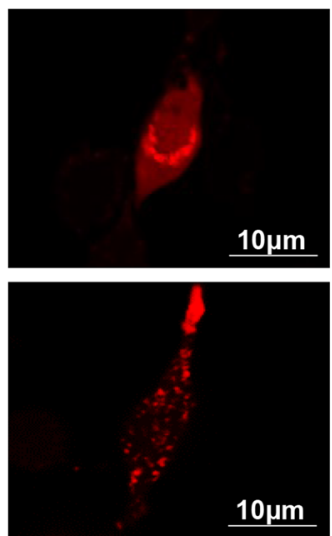

Merge
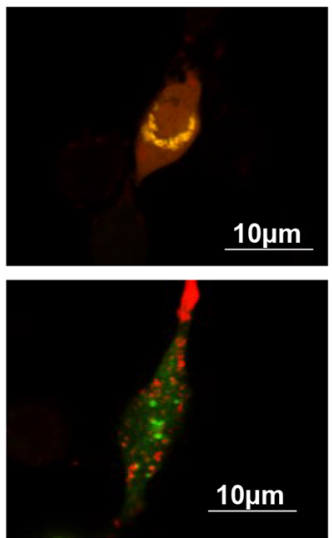

b

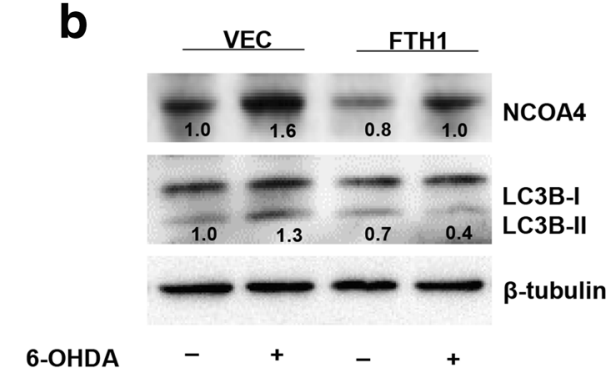

d

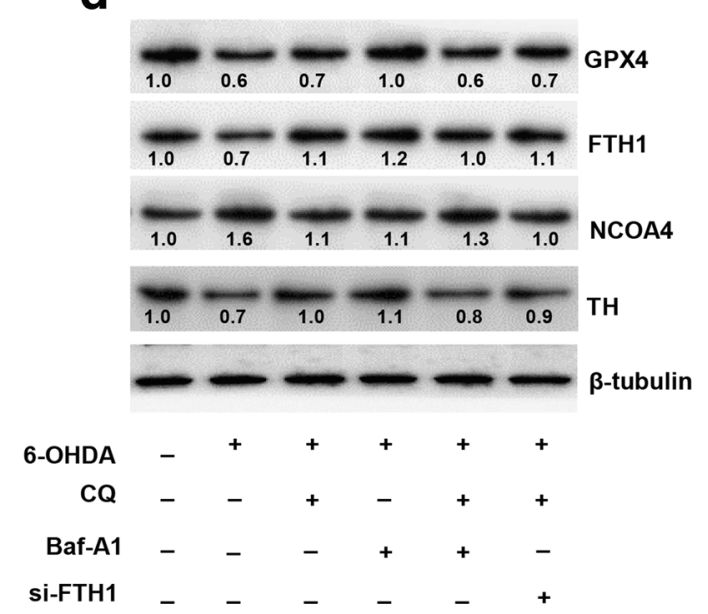

Fig. 10 FTH1 induced ferroptosis through ferritinophagy in the 6OHDA model of PD. The expression of NCOA4 and LC3B-I/ II in PC-12 cells transfected with si-FTH1 (A) or the overexpression lentivirus (B) was determined by Western blotting. The data were normalized to the control group values. (C) The effect of CQ $(20 \mu \mathrm{M})$ or Baf-A1 $(100 \mathrm{nM}) \mathrm{with} /$ without si-FTH1 was evaluated in PC-12 cells treated with $40 \mu \mathrm{M} 6-\mathrm{OHDA}$ for $24 \mathrm{~h}$. Cell viability was determined by the MTS assay. (D) The expression of GPX4, FTH1, NCOA4, and TH was determined by Western blotting. The data were normalized to the control group values a



C

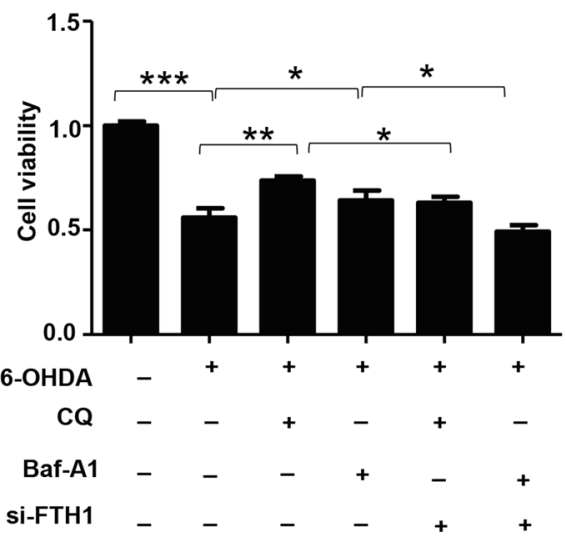


Fig. 11 FTH1 decreased

mitochondrial damage in PC-12

cells. Changes in mitochondrial morphology in PC-12 cells treated with si-FTH1 (A) or the over-

expression lentivirus (B) com-

bined with $40 \mu \mathrm{M}$ 6-OHDA were observed by transmission electron microscopy ( $n \geq 3$ assays) a

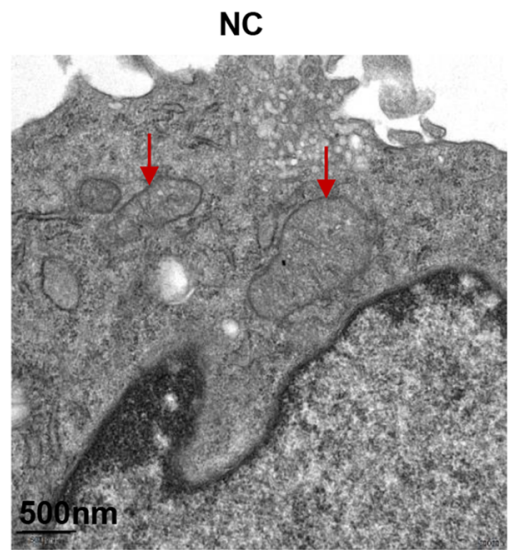

si-FTH1

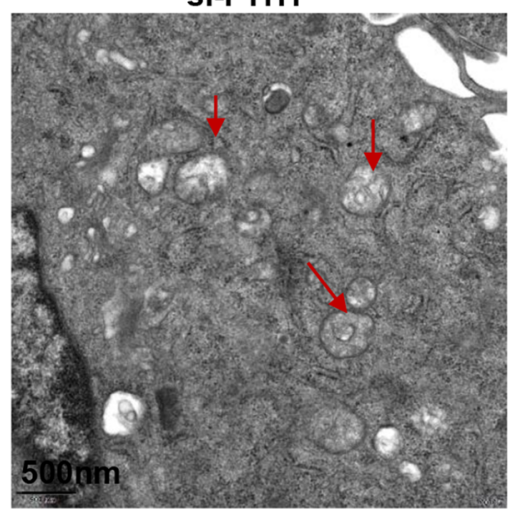

b

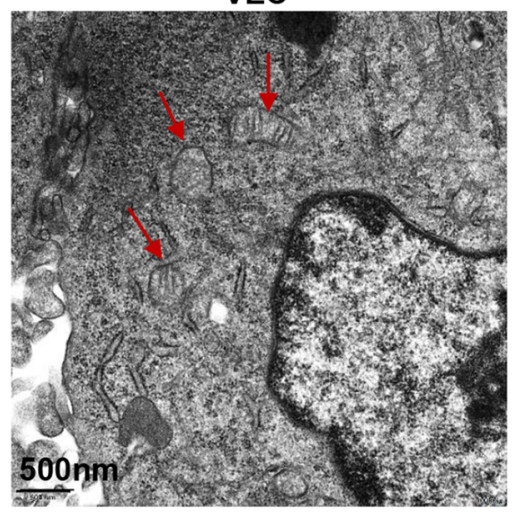

FTH1

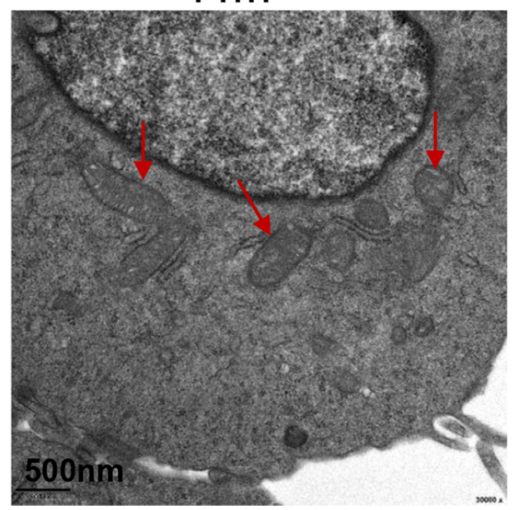

6-OHDA+NC

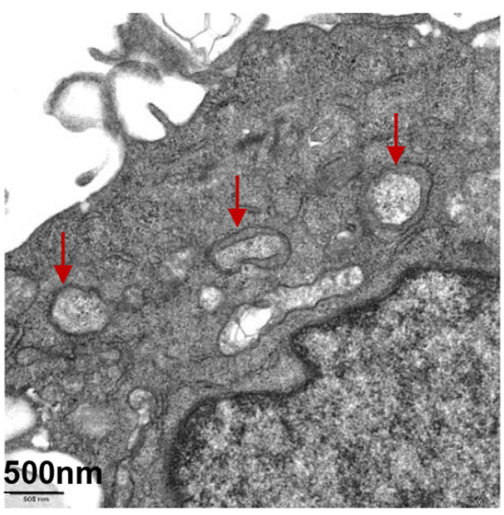

6-OHDA+si-FTH1

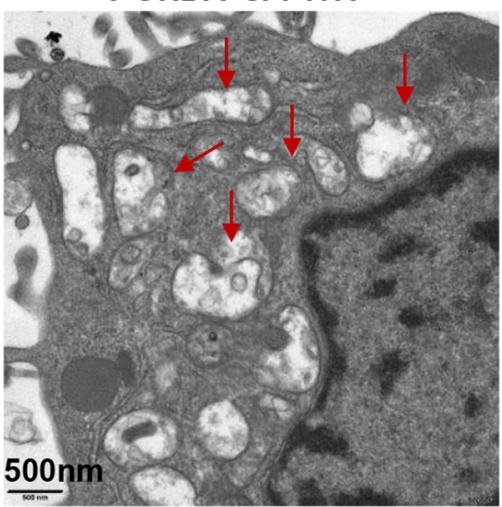

6-OHDA+VEC

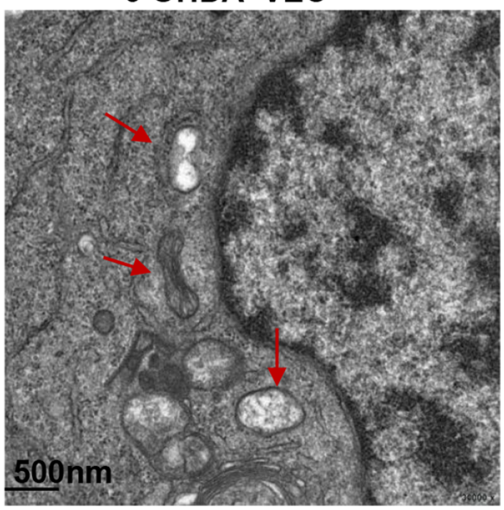

6-OHDA+FTH1

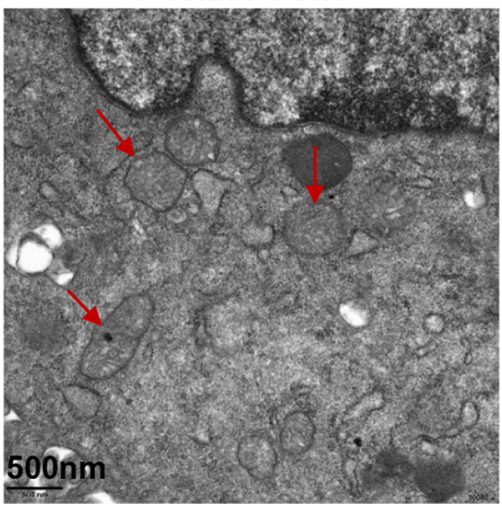


Fig. 12 Schematic diagram showing the mechanisms of FTH1. At a low level, FTH1 recruits $\mathrm{LC} 3$ and selectively binds to NCOA4 in autophagosomes, and then ferritinophagy is induced to degrade FTH1. After FTH1 is downregulated, it further promotes ferritinophagy. Eventually, FTH1 is maintained at an extremely low level, resulting in the accumulation of free ferrous ions and a reduction in the MMP and eventually leading to cell death due to ferroptosis in the 6-OHDA model of PD

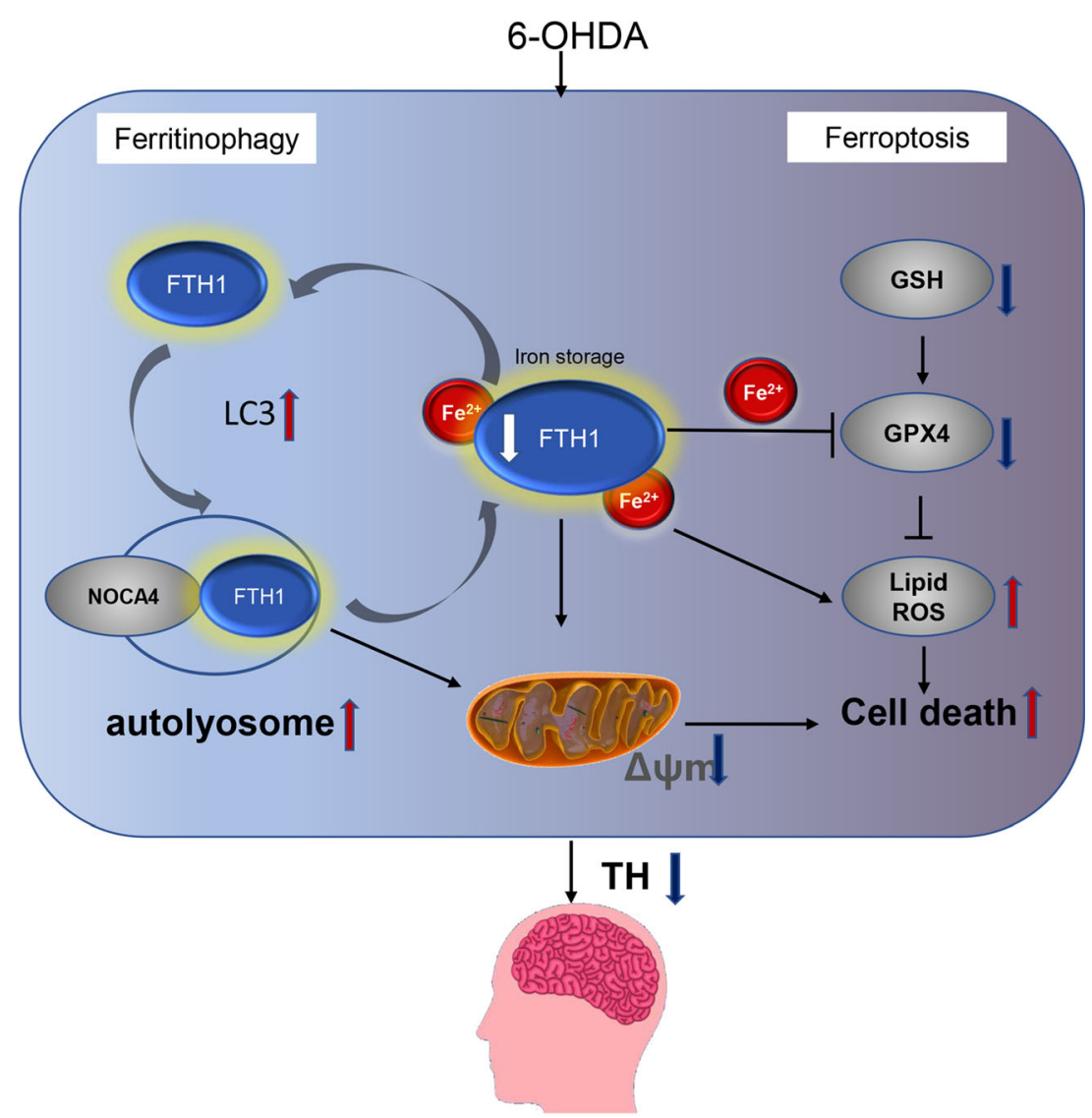

Parkinson's disease

\section{Discussion}

In the present study, we determined that FTH1 regulated cell ferroptosis via ferritinophagy in the 6-OHDA model of PD. Ferritinophagy is the autophagic degradation of the ferritin protein to maintain homeostasis during iron depletion. Previous studies have demonstrated that ferritinophagy is involved in various diseases. In a clinical trial on patients with diabetes and cardiovascular disease, phlebotomy-triggered ferritinophagy led to the release of redox-active iron and aggravated diabetes and cardiovascular disease [28]. The accumulation of intracellular iron and changes in iron homeostasis proteins were shown to occur in senescent cells. The results from previous studies demonstrate that ferritin degradation induced by lysosomal impairment is associated with impaired ferritinophagy and resistance to ferroptosis in mouse and human cell lines [29]. The RNA-binding protein ELAVL1/HuR activates ferritinophagy to regulate ferroptosis in hepatic stellate cells. ELAVL1-autophagy-dependent ferroptosis has been identified as a potential target for the treatment of liver fibrosis [30]. In addition, ferritinophagy is the molecular mechanism underlying free iron accumulation related to ferroptosis induction in chronic obstructive pulmonary disease pathogenesis [31]. Moreover, researchers have speculated that ferritinophagy provides a novel link between the levels of iron in the brain and neurodegenerative diseases [32]. Overexpression of $\alpha$-syn in cells impairs ferritinophagy by compromising lysosomal function, resulting in the accumulation of iron-rich ferritin in the outer retina in vivo and retinal pigment epithelial cells in vitro [33].

Ferritin is a widely expressed and highly conserved protein and consists of 2 types of polypeptide chains: ferritin heavy chain and ferritin light chain. Ferritin heavy chain catalyzes the $\mathrm{Fe}^{2+}$ oxidation reaction, whereas ferritin light chain plays an important role in the storage of $\mathrm{Fe}^{3+}$. Both chains are essential for maintaining iron homeostasis and preventing iron overload [34-36]. FTH1, a key subunit of ferritin, is involved in a variety of disease signaling pathways. In particular, the expression of FTH1 varies in different diseases. FTH1 was shown to regulate immunity in studies of prostate and breast cancer $[37,38]$. FTH1 has been shown to inhibit apoptosis through the JNK signaling pathway activity [39]. Sorafenib significantly decreases the expression of FTH1 during ferritinophagy activation in hepatic stellate cells [30]. FTH1 is reduced in liver fibrosis through regulation of the ferroptosis signaling pathway [40]. The activity of superoxide dismutase and brain iron levels are decreased in the brains of FTH1 knockout mice compared with those of control mice, indicating that FTH1 contributes to the disruption of iron homeostasis due to oxidative stress in the brain [41]. Knockdown of 
FTH1 in the intestines of mice can lead to iron overabsorption and promote ferroptosis $[42,43]$. The antioxidant effects of epicatechin are mediated by increased FTH1 expression. In addition, green tea catechins exert neuroprotective effects on iron metabolism by upregulating FTH1 expression [44]. Our studies are in agreement with the previous studies described above and provide further evidence to support the role of FTH1 as a promising target for neuroprotection.

However, several studies have shown opposite results. A study on peroxidation of polyunsaturated fatty acids showed that FTH1 expression increases during ferroptosis [45]. In addition, overexpression of FTH1 enhances ferritin degradation after induction of ferroptosis by erastin [18]. Remarkably, the protein level of FTH1 is significantly increased in MEFs and PANC1 cells after erastin treatment, suggesting that autophagy promotes ferroptosis by ferritin degradation [14]. LIP accumulation during ferroptosis induces transcriptional upregulation of endogenous FTH1 [15]. In general, autophagydriven ferroptosis occurs during the early initiation stage. As autophagy plays a complicated role in cell death and survival, FTH1 acts as the key protein in ferritinophagy and may have contradictory effects at different stages of disease. In summary, FTH1 may provide a key link between ferroptosis and ferritinophagy in disease. Regulating FTH1 may be therapeutically beneficial in the pathophysiology of PD.

Acknowledgments This work was supported by the Natural Science Foundation of Guangdong Province, China (Grant No. 2020A151501325), and the Administration of Traditional Chinese Medicine of Guangdong Province, China (Grant No. 20203010).

Required Author Forms Disclosure forms provided by the authors are available with the online version of this article.

\section{Compliance with Ethical Standards}

Conflict of Interest The authors declare that they have no competing interests.

\section{References}

1. Grayson M: Parkinson's disease. Nature 2016, 538(7626):S1.

2. Hayes MT: Parkinson's disease and parkinsonism. Am J Med 2019, 132(7):802-807.

3. Erkkinen MG, Kim MO, Geschwind MD: Clinical neurology and epidemiology of the major neurodegenerative diseases. Cold Spring Harb Perspect Biol 2018, 10(4).

4. Connolly BS, Lang AE: Pharmacological treatment of Parkinson disease: a review. JAMA 2014, 311(16):1670-1683.

5. Ward RJ, Zucca FA, Duyn JH, Crichton RR, Zecca L: The role of iron in brain ageing and neurodegenerative disorders. Lancet Neurol 2014, 13(10):1045-1060.

6. Moreau C, Duce JA, Rascol O, Devedjian JC, Berg D, Dexter D, Cabantchik ZI, Bush AI, Devos D, FAIRPARK-II Study Group: Iron as a therapeutic target for Parkinson's disease. Mov Disord 2018, 33(4):568-574.
7. Crielaard BJ, Lammers T, Rivella S: Targeting iron metabolism in drug discovery and delivery. Nat Rev Drug Discov 2017, 16(6): 400-423.

8. Healy S, McMahon JM, FitzGerald U: Modelling iron mismanagement in neurodegenerative disease in vitro: paradigms, pitfalls, possibilities \& practical considerations. Prog Neurobiol 2017, 158:114.

9. Guiney S, Adlard PA, Bush AI, Finkelstein DI, Ayton S: Ferroptosis and cell death mechanisms in Parkinson's disease. Neurochem Int 2017, 104 (2017) 34-48.

10. Do Van B, Gouel F, Jonneaux A, Timmerman K, Gele P, Petrault M, Bastide M, Laloux C, Moreau C, Bordet R et al: Ferroptosis, a newly characterized form of cell death in Parkinson's disease that is regulated by PKC. Neurobiol Dis 2016, 94:169-178.

11. Wu C, Zhao W, Yu J, Li S, Lin L, Chen X: Induction of ferroptosis and mitochondrial dysfunction by oxidative stress in PC12 cells. Sci Rep 2018, 8(1):574.

12. Xie Y, Hou W, Song X, Yu Y, Huang J, Sun X, Kang R, Tang D: Ferroptosis: process and function. Cell Death Differ 2016, 23(3): 369-379.

13. Dixon SJ, Lemberg KM, Lamprecht MR, Skouta R, Zaitsev EM, Gleason CE, Patel DN, Bauer AJ, Cantley AM, Yang WS et al.: Ferroptosis: an iron-dependent form of nonapoptotic cell death. Cell 2012, 149(5):1060-1072.

14. Hou W, Xie Y, Song X, Sun X, Lotze MT, Zeh HJ, 3rd, Kang R, Tang D: Autophagy promotes ferroptosis by degradation of ferritin. Autophagy 2016, 12(8):1425-1428.

15. Gao M, Monian P, Pan Q, Zhang W, Xiang J, Jiang X: Ferroptosis is an autophagic cell death process. Cell Res 2016, 26(9):10211032.

16. Muhoberac BB, Vidal R: Iron, ferritin, hereditary ferritinopathy, and neurodegeneration. Front Neurosci 2019, 13:1195.

17. Tang M, Chen Z, Wu D, Chen L: Ferritinophagy/ferroptosis: ironrelated newcomers in human diseases. J Cell Physiol 2018, 233(12):9179-9190.

18. Latunde-Dada GO: Ferroptosis: role of lipid peroxidation, iron and ferritinophagy. Biochim Biophys Acta, Gen Subj 2017, 1861(8): 1893-1900.

19. Mancias JD, Wang X, Gygi SP, Harper JW, Kimmelman AC: Quantitative proteomics identifies NCOA4 as the cargo receptor mediating ferritinophagy. Nature 2014, 509(7498):105-109.

20. Quiles Del Rey M, Mancias JD: NCOA4-mediated ferritinophagy: a potential link to neurodegeneration. Front Neurosci 2019, 13:238.

21. Santana-Codina N, Mancias JD: The role of NCOA4-mediated ferritinophagy in health and disease. Pharmaceuticals 2018, 11(4).

22. Doll S, Conrad M: Iron and ferroptosis: a still ill-defined liaison. IUBMB Life 2017, 69(6):423-434.

23. Lu J, Liu X, Tian Y, Li H, Ren Z, Liang S, Zhang G, Zhao C, Li X, Wang $\mathrm{T}$ et al: Moxibustion exerts a neuroprotective effect through antiferroptosis in Parkinson's disease. Evid Based Complement Alternat Med 2019, 2019:2735492.

24. Neitemeier S, Jelinek A, Laino V, Hoffmann L, Eisenbach I, Eying R, Ganjam GK, Dolga AM, Oppermann S, Culmsee C: BID links ferroptosis to mitochondrial cell death pathways. Redox Biol 2017, 12:558-570.

25. Abdalkader M, Lampinen R, Kanninen KM, Malm TM, Liddell JR: Targeting Nrf2 to suppress ferroptosis and mitochondrial dysfunction in neurodegeneration. Front Neurosci 2018, 12:466.

26. Skouta R, Dixon SJ, Wang J, Dunn DE, Orman M, Shimada K, Rosenberg PA, Lo DC, Weinberg JM, Linkermann A et al.: Ferrostatins inhibit oxidative lipid damage and cell death in diverse disease models. J Am Chem Soc 2014, 136(12):4551-4556.

27. Mauthe M, Orhon I, Rocchi C, Zhou X, Luhr M, Hijlkema KJ, Coppes RP, Engedal N, Mari M, Reggiori F: Chloroquine inhibits autophagic flux by decreasing autophagosome-lysosome fusion. Autophagy 2018, 14(8):1435-1455. 
28. Zacharski LR, Shamayeva G, Chow BK: Iron reduction response and demographic differences between diabetics and non-diabetics with cardiovascular disease entered into a controlled clinical trial. Metallomics 2018, 10(2):264-277.

29. Masaldan S, Clatworthy SAS, Gamell C, Meggyesy PM, Rigopoulos AT, Haupt S, Haupt Y, Denoyer D, Adlard PA, Bush AI et al.: Iron accumulation in senescent cells is coupled with impaired ferritinophagy and inhibition of ferroptosis. Redox Biol 2018, 14:100-115.

30. Zhang Z, Yao Z, Wang L, Ding H, Shao J, Chen A, Zhang F, Zheng $S$ : Activation of ferritinophagy is required for the RNA-binding protein ELAVL1/HuR to regulate ferroptosis in hepatic stellate cells. Autophagy 2018, 14(12):2083-2103.

31. Yoshida M, Minagawa S, Araya J, Sakamoto T, Hara H, Tsubouchi K, Hosaka Y, Ichikawa A, Saito N, Kadota T et al.: Involvement of cigarette smoke-induced epithelial cell ferroptosis in COPD pathogenesis. Nat Commun 2019, 10(1):3145.

32. Biasiotto G, Di Lorenzo D, Archetti S, Zanella I: Iron and neurodegeneration: is ferritinophagy the link? Mol Neurobiol 2016, 53(8):5542-5574.

33. Baksi S, Singh N: alpha-Synuclein impairs ferritinophagy in the retinal pigment epithelium: implications for retinal iron dyshomeostasis in Parkinson's disease. Sci Rep 2017, 7(1):12843.

34. Torti FM, Torti SV: Regulation of ferritin genes and protein. Blood 2002, 99(10):3505-3516.

35. Sammarco MC, Ditch S, Banerjee A, Grabczyk E: Ferritin L and H subunits are differentially regulated on a post-transcriptional level. J Biol Chem 2008, 283(8):4578-4587.

36. Friedman A, Arosio P, Finazzi D, Koziorowski D, GalazkaFriedman J: Ferritin as an important player in neurodegeneration. Parkinsonism Relat Disord 2011, 17(6):423-430.

37. Chan JJ, Kwok ZH, Chew XH, Zhang B, Liu C, Soong TW, Yang H, Tay Y: A FTH1 gene:pseudogene:microRNA network regulates tumorigenesis in prostate cancer. Nucleic Acids Res 2018, 46(4): 1998-2011.

38. Liu NQ, De Marchi T, Timmermans AM, Beekhof R, TrapmanJansen AM, Foekens R, Look MP, van Deurzen CH, Span PN,
Sweep FC et al.: Ferritin heavy chain in triple negative breast cancer: a favorable prognostic marker that relates to a cluster of differentiation 8 positive $(\mathrm{CD} 8+)$ effector T-cell response. Mol Cell Proteomics 2014, 13(7):1814-1827.

39. Liu F, Du ZY, He JL, Liu XQ, Yu QB, Wang YX: FTH1 binds to Daxx and inhibits Daxx-mediated cell apoptosis. Mol Biol Rep 2012, 39(2):873-879.

40. Kong Z, Liu R, Cheng Y: Artesunate alleviates liver fibrosis by regulating ferroptosis signaling pathway. Biomed Pharmacother 2019, 109:2043-2053.

41. Thompson K, Menzies S, Muckenthaler M, Torti FM, Wood T, Torti SV, Hentze MW, Beard J, Connor J: Mouse brains deficient in $\mathrm{H}$-ferritin have normal iron concentration but a protein profile of iron deficiency and increased evidence of oxidative stress. J Neurosci Res 2003, 71(1):46-63.

42. Tao Y, Wu Q, Guo X, Zhang Z, Shen Y, Wang F: MBD5 regulates iron metabolism via methylation-independent genomic targeting of Fth1 through KAT2A in mice. Br J Haematol 2014, 166(2):279291.

43. Sun X, Ou Z, Chen R, Niu X, Chen D, Kang R, Tang D: Activation of the p62-Keap1-NRF2 pathway protects against ferroptosis in hepatocellular carcinoma cells. Hepatology 2016, 63(1):173-184.

44. Chiba T, Fujita S, Kubota H, Inoue D, Mizuno A, Komatsu T, Yamaza H, Higami Y, Shimokawa I: Identification of fastinginduced genes in the rat hypothalamus: relationship with neuroprotection. Ann N Y Acad Sci 2007, 1119:216-226.

45. Yamada N, Karasawa T, Kimura H, Watanabe S, Komada T, Kamata R, Sampilvanjil A, Ito J, Nakagawa K, Kuwata H et al.: Ferroptosis driven by radical oxidation of $n-6$ polyunsaturated fatty acids mediates acetaminophen-induced acute liver failure. Cell Death Dis 2020, 11(2):144.

Publisher's Note Springer Nature remains neutral with regard to jurisdictional claims in published maps and institutional affiliations. 